\title{
Quasi-convexity and shrinkwrapping
}

\author{
HOSSEIN NAMAZI
}

\begin{abstract}
We extend a result of Minsky to show that, for a map of a surface to a hyperbolic 3-manifold, which is 2-incompressible rel a geodesic link with a definite tube radius, the set of noncontractible simple loops with bounded length representatives is quasiconvex in the complex of curves of the surface. We also show how wide product regions can be used to find a geodesic link with a definite tube radius with respect to which a map is 2-incompressible.
\end{abstract}

57M50; 57N10, 30F40

\section{Introduction}

Suppose $S$ is a closed orientable surface of genus $\geq 2$. The complex of curves of $S$, denoted by $\mathcal{C}(S)$, is a complex whose vertices are homotopy classes of noncontractible simple loops on $S$. Two vertices are connected by an edge if they have disjoint representatives on the surface. Using this we endow $\mathcal{C}(S)$ with a geodesic metric where the length of each edge is one. One of our motivations in this article is the following question:

Question Given a surface $S$, do there exist constants $B, K>0$ such that, for every irreducible representation $\rho: \pi_{1}(S) \rightarrow \mathrm{PSL}_{2}(\mathbb{C})$, the $\operatorname{set} \mathcal{C}(B, \rho)$ is a $K$-quasi-convex subset of $\mathcal{C}(S)$ ? Here $\mathcal{C}(B, \rho) \subset \mathcal{C}(S)$ denotes the homotopy classes of simple loops $\alpha$ on $S$, where the translation length of $\rho(\alpha)$ is at most $B$.

Recall that for a geodesic metric space $X$, a subset $Y \subset X$ is $K$-quasi-convex if for every two points $p, q \in Y$, every geodesic connecting $p$ and $q$ is contained in the $K$-neighborhood of $Y$. Also note that, even though a closed curve $\alpha$ only identifies a conjugacy class in $\pi_{1}(S)$, it makes sense to speak of the translation length of $\rho(\alpha)$ because the translation length stays invariant under conjugacy.

We do not know any case where the answer to the above question is negative. Our result lies somewhere between two cases where partial affirmative answers are known. These results have been significant in relating the topology and geometry of 3-manifolds to the combinatorics of curves on a surface. The first result is a theorem of Minsky 
[16] which has been a central theme in the construction of models for hyperbolic 3 -manifolds and the proof of Thurston's Ending Lamination Conjecture by Brock, Canary and Minsky [15;2]. Suppose $j: S \rightarrow N$ is a $\pi_{1}$-injective map from a closed orientable surface $S$ of genus $>1$ into the hyperbolic 3 -manifold $N$ and assume $\mathcal{C}(B, j)$ denotes the homotopy classes of noncontractible simple loops $\alpha$ where $j(\alpha)$ has a representative of length $\leq B$ in $N$. For $B$ large enough depending on $\chi(S)$, the set $\mathcal{C}(B, j)$ is quasi-convex in the complex of curves of $S$, with a quasi-convexity constant that depends only on $\chi(S)$ and $B$.

The second result is more topological and considers the case when $M$ is a compact 3-manifold and $S$ is a compressible component of $\partial M$. Then Masur and Minsky [13] show that the homotopy classes of noncontractible simple loops on $S$ which are compressible in $M$ form a quasi-convex subset of the complex of curves of $S$. Here the quasi-convexity constant depends only on $\chi(S)$. In particular for every faithful representation of $\pi_{1}(M)$, if we let $\rho$ be the representation of $\pi_{1}(S)$ given by composition with the homomorphism induced by the inclusion $S \hookrightarrow M$, the set $\mathcal{C}(B, \rho)$ is quasi-convex for $B=0$. Note that, in contrast to the previous mentioned result, in this case the representation is not faithful.

The above question has been motivated mostly by the first result and the major role that it plays in the study and description of the geometry of hyperbolic 3-manifolds. Complete or partial answers to the question then become important steps in understanding how many of these results it is possiblle to carry out in a wider setting. Also compare with work of Bowditch in [1], where he considers "type preserving" representations of the fundamental group of a punctured torus. Note that, for the punctured torus, even though the surface is not closed, we can define the complex of curves by letting the vertices be the homotopy classes of nonperipheral noncontractible simple loops and by connecting a pair of vertices if they have representatives that intersect once. In this case, it is easy to see that this complex is nothing other than the Farey graph. In [1], it is shown that for a type preserving representation, the set $\mathcal{C}(B, j)$ gives a connected subset of the Farey graph. In this case, Bowditch's argument can be used to show that $\mathcal{C}(B, j)$ is also 1-quasi-convex.

We extend Minsky's result in [16] by replacing the condition that $j$ is $\pi_{1}$-injective with a weaker condition. Given a hyperbolic 3 -manifold $N$, we say a geodesic link $\Gamma \subset N$ is $\eta$-separated for $\eta>0$ if $\Gamma$ has an embedded tubular neighborhood of radius $\eta$. Suppose $N$ has no parabolics, $S$ is orientable and $j: S \rightarrow N$ is 2-incompressible with respect to an $\eta$-separated geodesic link $\Gamma \subset N$. By 2-incompressibility of $j$ (in the case $S$ is a closed surface and $N$ has no parabolics) we mean it is $\pi_{1}-$ injective as a map to $N \backslash \Gamma$ and the image of a noncontractible loop cannot be freely homotopic to a (multiplied) meridian of a component of $\Gamma$. Finally for a loop $\gamma$ on $S$, 
a representative in $N \backslash \Gamma$ is a closed curve that is freely homotopic to $j(\gamma)$ in $N \backslash \Gamma$. The set $\mathcal{C}(B, j) \subset \mathcal{C}(S)$ consists of noncontractible simple loops with a representative of length $\leq B$ in $N \backslash \Gamma$.

Theorem 1.1 Suppose $N$ is an oriented hyperbolic 3-manifold, $\Gamma \subset N$ is an $\eta-$ separated geodesic link and $j: S \rightarrow N$ is 2 -incompressible rel $\Gamma$. Then for $B$ sufficiently large, the set $\mathcal{C}(B, j)$ is $K$-quasi-convex for a constant $K$ that depends only on $\chi(S), \eta$ and $B$.

This is obviously stronger than Minsky's result. The assumption on the map $j$ is significantly weakened; in particular the map $j$ does not have to be $\pi_{1}$-injective as a map into $N$. Another advantage of our proof is that the constant $K$ is made more effective and is computable as a function of $\chi(S), \eta$ and $B$. In Theorem 4.7 we give the precise statement of the above theorem, which also includes the more general cases when $S$ does not have to be closed and $N$ may have parabolics.

In applications, one needs to guarantee existence of $\eta$-separated geodesic links which make an embedded surface 2-incompressible. We show how one can obtain such geodesic links by using "wide product regions." We prove a stronger result that provides the 2-incompressibility for the embedding of a compact 3-manifold in a hyperbolic 3-manifold. Given a compact oriented 3-manifold $M$ and an embedding $j: M \hookrightarrow N$ into an oriented hyperbolic 3-manifold $N$ without parabolics, we say $j$ is 2-incompressible rel a geodesic link $\Gamma \subset N \backslash j(M)$, if $j$ is $\pi_{1}$-injective as a map from $M$ to $N \backslash \Gamma$ and the image of a noncontractible loop in $M$ is not homotopic to a (multiplied) meridian of a component of $\Gamma$. Note that an embedding of a surface is 2-incompressible if and only if the embedding of a collared neighborhood of the surface is 2-incompressible.

Given a closed oriented surface $E$ of genus $\geq 2$ and a hyperbolic 3 -manifold $N$, a product region is a submanifold of $N$ homeomorphic to $E \times[0,1]$. A level surface of the product region is a surface that is isotopic to $E \times\{0\}$ within the product region. A "standard" product region is a product region in a doubly degenerate hyperbolic structure on $E \times \mathbb{R}$ which is enclosed by two surfaces in small neighborhoods of "simplicial hyperbolic surfaces." In a hyperbolic 3-manifold, when we speak about a product region we mean a product region that is $L$-bilipschitz to a standard product region; sometimes we refer to these as $L$-product regions. Given a constant $\epsilon_{0}$, typically smaller than the Margulis constant in dimension 3, we define the width of a product region in $N$ to be the distance in the product region between its boundary components relative to the $\epsilon_{0}$-thin components of $N$. In Section 5 , we define these notions precisely and state a more general and rigorous version of the following theorem 
which generalizes the result to the case when we have a proper embedding of a pared manifold and $N$ is allowed to have parabolics.

Theorem 1.2 Let $M$ be an irreducible compact 3-manifold with no torus boundaries and $j: M \rightarrow N$ an embedding into an oriented hyperbolic 3-manifold $N$ without parabolics such that the $j$-image of every component of $\partial M$ is a level surface of a product region with sufficiently large width. Then there exists a 0.025 -separated geodesic link $\Gamma$ in the product regions of components of $\partial M$ such that $j$ is 2 -incompressible rel $\Gamma$.

Obviously the definition of the product region and width involve the constants $L$ and $\epsilon_{0}$; the required width in the conclusion will be explicit and depends on $L, \epsilon$ and $\chi(\partial M)$. An important case of the above theorem is when $M=S \times I$ is a trivial bundle over a surface. As we mentioned earlier, in this case 2-incompressibility of an embedding of $M$ is equivalent to the 2-incompressibility of the restriction of the embedding to $S \times\{0\}$. In this case we prove Theorem 5.3 which in particular implies:

Theorem 1.3 Suppose $S$ is a closed oriented surface with negative Euler characteristic and $j: S \rightarrow N$ is an embedding into an oriented hyperbolic 3-manifold $N$ without parabolics such that to each side $j(S)$ is either incompressible or is a level surface of a product region with sufficiently large width. Then there exists a 0.025 -separated geodesic link in the product regions such that $j(S)$ is 2-incompressible rel $\Gamma$.

Note that since $S$ and $N$ are both oriented, $j(S)$ is two-sided. We say $j(S)$ is incompressible to one side if for every map $D^{2} \rightarrow N$ whose image intersects $j(S)$ in the image of $\partial D^{2}$ and the image of a regular neighborhood of $\partial D^{2}$ is positioned on that side, one can conclude that the image of $\partial D^{2}$ is contractible on $j(S)$.

The main application of the above theorems is in construction of models and interpolations. This is obviously an extension of similar application for Minsky's quasi-convexity results in $[16 ; 15]$. The advantage of our approach is to find local models by using weaker assumptions.

In [17] our results here are used to obtain models for a class of hyperbolic structures on handlebodies and then these models are used to construct nearly hyperbolic negatively curved metrics on a class of closed 3-manifolds. In this application and also in the joint work with Brock, Minsky and Souto [3], one is dealing with a map of a surface to a hyperbolic 3-manifold which is not $\pi_{1}$-injective, for example when $S$ is a Heegaard surface; but using other features of the geometry, one can find $\eta$-separated geodesic links which make the surface 2 -incompressible. This is usually done by constructing 
wide product regions and applying Theorem 1.3. Then Theorem 1.1 can be used successfully. A main ingredient of the proofs is a use of shrinkwrapped surfaces. The original construction of such maps was developed in work of Calegari and Gabai in the proof of the Tameness Conjecture. We, however, rely more on softer constructions of such surfaces more similar to the work of Soma [19]. In fact, we go one step further than Soma's construction and use those arguments to construct simplicial hyperbolic surfaces in the sense of Thurston and Canary. We explain the construction of these surfaces and the main properties of them in the presence of $\eta$-separated geodesic links in Section 3. Then in Section 4, we use these surfaces to prove a generalization of Theorem 1.1. We basically reconstruct Minsky's argument in [16] by using our simplicial hyperbolic surfaces. We define a projection $\Pi_{j, B}$ from $\mathcal{C}(S)$ to $\mathcal{C}(B, j)$ and in Proposition 4.5, we prove that this projection behaves almost like the closest point projection onto a convex set in a hyperbolic space. Then a standard argument of Minsky proves the image $\mathcal{C}(B, j)$ is quasi-convex. We should point out that in the proof of these properties in Lemma 4.4, we prove a weak version of Thurston's Uniform Injectivity Theorem for the simplicial hyperbolic surfaces. Our proof is an elementary case of the proof of the Uniform Injectivity Theorem given in Namazi and Souto [18]. This is one of the main differences with Minsky's proof, and the constructive nature of this proof allows a computation of an upper bound for the quasi-convexity constant.

Finally in Section 5, we prove a generalized version of Theorem 1.3. We use the wide product region to find a collection of geodesic closed curves with large pairwise distance. Then we use an argument of Gabai [8] to deform these into a collection $\Gamma$ in a way that the length decreases and eventually, they have a definite tube radius. Then by means of contradiction, we assume $j: S \rightarrow N$ is not 2-incompressible rel $\Gamma$ and perform compressions and surgeries that each intersect $\Gamma$ at most once. When we are done, the obtained surfaces $S_{1}, \ldots, S_{r}$ are 2-incompressible with respect to a subset $\Gamma_{1}$ of $\Gamma$ that has not been involved in the process. When the number of components of $\Gamma$ is large enough then the number of components of $\Gamma_{1}$ is also large, and we use this to show for one of the surfaces $S_{i}$, we must have $\chi\left(S_{i}\right) \leq \chi(S)$ which is a contradiction. This argument owes a huge debt to the argument of Calegari and Gabai in [5] and follows their ideas. Finally we use this argument and a topological observation to prove a generalized version of Theorem 1.2.

The author is grateful to Yair Minsky, David Gabai and Juan Souto for their encouragement and very useful conversations regarding this work. He is also grateful to the referee for many useful comments and in particular for pointing out some simplifications in the construction of the simplicial hyperbolic surfaces and also for suggesting the current generalized statement of Theorem 1.2. 


\section{Preliminaries}

\subsection{Hyperbolic geometry}

A hyperbolic manifold is a complete Riemannian manifold with constant sectional curvature -1 . In this article, we only consider hyperbolic manifolds with finitely generated fundamental group. Recall that in a Riemannian manifold $N$, the injectivity radius of $N$ at a point $x \in N$, denoted by $\operatorname{inj}_{N}(x)$, is half the length of the shortest (homotopically nontrivial) loop through $x$. We use the notation $N \leq \epsilon$ (resp. $N^{<\epsilon}, N^{\geq \epsilon}, N^{>\epsilon}$ ) to denote the set of points $x \in N$ where $\operatorname{inj}_{N}(x) \leq \epsilon$ (resp. $\left.\operatorname{inj}_{N}(x)<\epsilon, \operatorname{inj}_{N}(x) \geq \epsilon, \operatorname{inj}_{N}(x)>\epsilon\right)$. By the Margulis lemma, there exists a universal constant $\epsilon_{M}>0$, such that in a hyperbolic 3-manifold $N$ and for every $\epsilon \leq \epsilon_{M}$, every component of $N^{\leq \epsilon}$, the $\epsilon$-thin part of $N$, is either

(1) a rank two cusp: a horoball in $\mathbb{H}^{3}$ modulo a parabolic action of $\mathbb{Z} \oplus \mathbb{Z}$,

(2) a rank one cusp: a horoball in $\mathbb{H}^{3}$ modulo a parabolic action of $\mathbb{Z}$ or

(3) a solid torus neighborhood of a closed geodesic.

The components of type (1) and (2) above are called cusps of $N$ and a component $T$ of type (3) is called a Margulis tube. A Margulis tube $T$ is a solid torus neighborhood of a closed geodesic $\gamma_{T}$ of length $\leq \epsilon$, which we call the core of the Margulis tube. We also define $N_{\epsilon}$ to denote the manifold obtained by removing the interiors of all the $\epsilon-$ thin cusps of $N$.

Given $\epsilon \leq \epsilon_{M}$ and $x, y \in N_{\epsilon}$, we define

$$
d_{N}^{\geq \epsilon}(x, y)
$$

to be the minimum of $l_{N}\left(\kappa \cap N^{\geq \epsilon}\right)$ among rectifiable paths $\kappa \subset N_{\epsilon}$ which connect $x$ and $y$. Here $l_{N}\left(\kappa \cap N^{\geq \epsilon}\right)$ is the total length of components of $\kappa \cap N^{\geq \epsilon}$ and we are insisting on considering only those paths that avoid the $\epsilon$-thin cusps of $N$, ie are subsets of $N_{\epsilon}$. This is different from the definition of $d^{\geq \epsilon}$ in most of the literature. Sometime, we refer to $d_{N}^{\geq \epsilon}(x, y)$ as the distance in $N_{\epsilon}$ between $x$ and $y$ rel $N^{\geq \epsilon}$. Similarly for $A \subset N$ we define $\operatorname{diam}_{N} \geq \epsilon(A)$ to be the supremum of $d_{N}^{\geq \epsilon}(x, y)$ for points $x, y \in A \cap N_{\epsilon}$ and for two subsets $A_{1}, A_{2} \subset N, d_{N}^{\geq \epsilon}\left(A_{1}, A_{2}\right)$ denotes the infimum of $d_{N}^{\geq \epsilon}(x, y)$ for points $x \in A_{1} \cap N_{\epsilon}, y \in A_{2} \cap N_{\epsilon}$.

We will need the following lemma in our arguments. It is an elementary application of the fact that projection to a convex set in the hyperbolic space contracts distances by a factor of $\cosh r$ where $r$ is the distance to the convex set. 
Lemma 2.1 Let $N$ be a hyperbolic 3-manifold and $\alpha$ a homotopically nontrivial closed curve in $N$ and $\alpha^{*}$ its geodesic representative. Then

$$
\cosh d_{N}\left(\alpha, \alpha^{*}\right) \leq l_{N}(\alpha) / l_{N}\left(\alpha^{*}\right),
$$

where $l_{N}(\alpha)$ is the length of $\alpha$ as a curve in $N$. Also given $\epsilon>0$

$$
\cosh d_{N}^{\leq \epsilon}\left(\alpha, \alpha^{*}\right) \leq l_{N}(\alpha) / \epsilon,
$$

where $d_{N}^{\leq \epsilon}$ denotes the distance rel $N \leq \epsilon$.

In addition, we need the next lemma to give us a bound for the distance between boundaries of nested components of $N^{\leq \epsilon_{1}}$ and $N \leq \epsilon_{2}$ for $\epsilon_{1} \neq \epsilon_{2}$ smaller than the Margulis constant. We refer the reader to Brooks and Matelski [4] and Meyerhoff [14] for a discussion and computation of the constants.

Lemma 2.2 There exists a homeomorphism $\xi:[1, \infty) \rightarrow[0, \infty)$ such that for every hyperbolic 3-manifold $N$ and $\epsilon_{2}<\epsilon_{1}$ both smaller than the Margulis constant

$$
d_{N}\left(N^{\leq \epsilon_{2}}, N^{\geq \epsilon_{1}}\right) \geq \xi\left(\epsilon_{1} / \epsilon_{2}\right) .
$$

In particular, using the above lemma, we assume the Margulis constant $\epsilon_{M}$ is chosen such that the distance between every two distinct components of $N \leq \epsilon_{M}$ is at least 1 .

A complete hyperbolic metric on the interior of a compact 3-manifold $M$ canonically corresponds to the conjugacy class of a discrete and faithful representation $\rho: \pi_{1}(M) \rightarrow$ Isom $\left(\mathbb{H}^{3}\right)$. The image $\rho\left(\pi_{1}(M)\right)$ of this representation is a Kleinian group and we can consider the limit set of its action which is a closed subset $\Lambda$ of the sphere at infinity. The convex hull of $\Lambda$ is invariant under the action of $\rho\left(\pi_{1}(M)\right)$ and its projection to the hyperbolic 3-manifold is called the convex core. When $M=S \times[0,1]$ and the limit set of $\rho\left(\pi_{1}(M)\right)$ is the entire sphere at infinity, we say the hyperbolic structure is doubly degenerate.

\subsection{Complex of curves}

Suppose $\bar{S}=S_{g, b}$ is a compact oriented surface of finite type with genus $g$ and $b$ boundary components and we always assume $\chi(\bar{S})<0$. A closed curve is nonperipheral if it is noncontractible and is not freely homotopic to a multiple of a peripheral closed curve on $\bar{S}$. Also if $\alpha$ is a properly embedded arc in $\bar{S}$, we say $\alpha$ is nonperipheral if $\alpha$ is not homotopic rel endpoints to an arc contained in $\partial \bar{S}$. Two nonperipheral curves (resp. arcs) are parallel if they are homotopic (resp. homotopic rel $\partial \bar{S}$ ). 
The complex of curves $\mathcal{C}(S)$ of $\bar{S}$ is a complex where every vertex is either a homotopy class of a nonperipheral simple loop or is the homotopy class (rel $\partial \bar{S}$ ) of a nonperipheral properly embedded arc. A subset $\left\{v_{0}, \ldots, v_{n}\right\}$ of distinct vertices form an $n$-simplex if these homotopy classes have representatives on $\bar{S}$ which do not intersect. We refer to such a set $\left\{v_{0}, \ldots, v_{n}\right\}$ or to a representation of that on $S$ as a multiarc. We equip $\mathcal{C}_{1}(S)$, the one-skeleton of $\mathcal{C}(S)$, with a path metric such that the length of every edge is equal to one. It is not hard to extend this metric to $\mathcal{C}(S)$ but the obtained metric space will be quasi-isometric to its one-skeleton and therefore for our purposes, it makes sense to treat $\mathcal{C}(S)$ without distinguishing it from its 1 -skeleton. We need also to point out that our definition of the complex of curves is slightly different from the definition in most of the literature and what we have defined is referred to as the complex of curves and arcs. However it is elementary to see the two complexes are quasi-isometric and being interested only in the large scale geometry of these complexes, we ignore the difference.

Masur and Minsky [11] proved that if $\bar{S}$ has negative Euler characteristic and is not the 3-holed sphere $S_{0,3}$ then $\mathcal{C}(S)$ has infinite diameter and is hyperbolic in sense of Gromov.

\section{Shrinkwrapping}

A geodesic link $\Gamma$ in a hyperbolic 3-manifold $N$ is a disjoint union of finitely many simple closed geodesics in $N$. A $\Gamma$-homotopy is a homotopy $F: X \times[0,1] \rightarrow N$ such that if $F(x, 0) \notin \Gamma$ then $f(x, t) \notin \Gamma$ for $t<1$ and if $f(x, 0) \in \Gamma$ then $f(x, t)=f(x, 0)$ for all $t \in[0,1]$. We say $X_{1}=F(X \times\{1\})$ is $\Gamma$-homotopic to $X_{0}=F(X \times\{0\})$.

Let $S$ be the interior of a compact surface $\bar{S}$ of finite type. A proper continuous map $j: S \rightarrow N$ is said to be 2-incompressible rel a geodesic link $\Gamma$ if

(a) $j(S) \cap \Gamma=\varnothing$, and $j_{*}: \pi_{1}(S) \rightarrow \pi_{1}(N \backslash \Gamma)$ is injective,

(b) the $j$-image of a noncontractible loop is not freely homotopic in $N \backslash \Gamma$ to a (multiplied) meridian of any component of $\Gamma$ and

(c) given a neighborhood $U$ of a cusp of $N$ such that $U \cap \Gamma=\varnothing$, the $j$-image of a nonperipheral simple loop is not freely homotopic in $N \backslash \Gamma$ into $U$.

Before we continue with our constructions, let us point out where our arguments differ from Soma's construction in [19] and how one could use Soma's arguments instead. Suppose $\Gamma$ is a geodesic link and $j: S \rightarrow N$ is 2 -incompressible rel $\Gamma$. The universal cover of $N \backslash \Gamma$ is a simply connected incomplete hyperbolic 3-manifold $\widetilde{N \backslash \Gamma}$. 
Then $X$, the metric completion of the cover, is the same except for an addition of a geodesic line for every lift of a tubular neighborhood of a component of $\Gamma$. The projection from the universal cover to $N \backslash \Gamma$ extends to a projection $X \rightarrow N$ and restricted to a small neighborhood of every added line, the projection is an infinite cyclic branch covering branched over a component of $\Gamma$. In this situation Soma shows that $X$ is CAT $(-1)$. The action of $\pi_{1}(N \backslash \Gamma)$ as the group of deck transformations on $\overparen{N \backslash \Gamma}$ extends to an action by isometries on $X$. Let $Y$ be the quotient of $X$ by the action of the subgroup which is the image of $\pi_{1}(S)$ by the map induced by $j$ on the level of fundamental groups. Then $j$ lifts to a map $\tilde{j}: S \rightarrow Y$. Even more it follows from condition (b) and (c) in the definition of 2-incompressibility that the projection $Y \rightarrow N$ is an infinite cyclic branch cover branched over a component of $\Gamma$, when restricted to the preimage of a small neighborhood of a component of $\Gamma$, and also that $\tilde{j}$ is $\pi_{1}$-injective. Given this, one can find alternatives to our results in this section where instead of working with $\Gamma$-geodesics and $\Gamma$-homotopies, everything is lifted to $Y$ and one simply takes geodesics in $Y$. Here we have decided not to refer to these infinite branched covers and prove everything in $N$.

Another difference with Soma's work [19] is that, he constructs and uses CAT(-1)piecewise ruled surfaces in the metric completion of the infinite branched covers. In our construction in Theorem 3.7, we have ruled surfaces in an intermediate stage but soon we turn them into more familiar simplicial hyperbolic surfaces. This is motivated by Gabai's notes [9].

It follows from an argument of Kerckhoff (cf Kojima [10]) that given a geodesic link $\Gamma$ in a hyperbolic 3-manifold $N$ and $t>0$ sufficiently small, there is a continuous family of complete negatively curve metrics $g_{t}$ on $N \backslash \Gamma$ that $g_{t}$ agrees with the hyperbolic metric on $N$ in the complement of $\mathcal{N}(\Gamma, t)$, the $t$-neighborhood of $\Gamma$. As a result we have the following lemma, which will be used later, and shows that the only $\pi_{1}$-injective immersed tori in $N \backslash \Gamma$ are the peripheral ones.

Lemma 3.1 Suppose $\Gamma$ is a geodesic link in the hyperbolic 3-manifold $N$ and $f: S^{1} \times S^{1} \rightarrow N \backslash \Gamma$ then one of the following happens:

- The image of the induced map on the fundamental group is cyclic.

- Within $N \backslash \Gamma, f$ is homotopic into a tubular neighborhood of a component of $\Gamma$.

- Within $N \backslash \Gamma, f$ is homotopic into a neighborhood of a rank 2 cusp of $N$ that does not intersect $\Gamma$.

In what follows by a path $\alpha \subset N$, we mean a continuous map $\alpha:(0,1) \rightarrow N$ with a lift $\tilde{\alpha}:(0,1) \rightarrow \mathbb{H}^{3}$ to the universal cover of $N$, such that $\lim _{t \rightarrow 0} \tilde{\alpha}(t)$ and $\lim _{t \rightarrow 1} \tilde{\alpha}(t)$ 
exist in the natural compactification of $\mathbb{H}^{3}$. An endpoint of the path is a projection of any of the above limits which is finite and an ideal endpoint of the path is any of the above limits that belongs to $\partial \mathbb{H}^{3}$. To avoid further complications, we only allow paths with ideal endpoints that are fixed points of parabolic fixed points of $\pi_{1}(N)$. In particular, the projection to $N$ of a path with ideal endpoints is always proper.

We say $\alpha_{0}, \alpha_{1} \subset N$ are homotopic (resp. $\Gamma$-homotopic) rel. endpoints if there is a homotopy (resp. $\Gamma$-homotopy) $F:(0,1) \times[0,1] \rightarrow N$ between $\alpha_{0}$ and $\alpha_{1}$ such that $\alpha_{s}(\cdot)=F(\cdot, s)$ has the same endpoints and ideal endpoints as $\alpha_{0}$, for $0 \leq s \leq 1$. We say a loop in $N \backslash \Gamma$ is nonperipheral if it is essential in $N \backslash \Gamma$ and cannot be homotoped by a $\Gamma$-homotopy into a neighborhood of a cusp of $N$ that does not intersect $\Gamma$. A path in $N \backslash \Gamma$ is nonperipheral if either its endpoints are not the same ideal points or it cannot be homotoped (within $N \backslash \Gamma$ into some small neighborhood of the cusp associated to its endpoints.

Lemma 3.2 [9; 19] If $\alpha_{0} \subset N \backslash \Gamma$ is a nonperipheral path or a loop, which is not freely homotopic (in $N \backslash \Gamma$ ) to a multiplied meridian of a component of $\Gamma$, there exists a unique piecewise geodesic $\alpha_{1}, \Gamma$-homotopic (rel endpoints if $\alpha_{0}$ is a path) to $\alpha_{0}$, such that $\alpha_{1}$ is length minimizing among all paths $\Gamma$-homotopic (rel endpoints) to $\alpha_{0}$. The function that takes $\alpha_{0}$ to $\alpha_{1}$ is continuous.

The proof is a simple generalization of the argument in [9] and we only provide a sketch of the proof.

Proof In the beginning assume $\alpha_{0}$ is either a loop or is a path with finite endpoints in $N \backslash \Gamma$. As mentioned earlier, there is a continuous family of complete negatively curved metrics $g_{t}$ on $N \backslash \Gamma$, such that $g_{t}$ agrees with the hyperbolic metric $g_{0}$ on $N$ outside of the complement of $\mathcal{N}(\Gamma, t)$. By the Cartan-Hadamard theorem, the conclusion holds for the $g_{t}$-metric. A limiting argument shows that the same holds for $g_{0}$. Even when $\alpha_{0}$ is a path whose endpoints are possibly ideal or are on $\Gamma$, one can use another limiting argument and show that the conclusion holds.

A piecewise geodesic $\alpha_{1}$ obtained in the conclusion of the above lemma is called a $\Gamma$-geodesic. The uniqueness of $\Gamma$-geodesics in their homotopy class (rel endpoints if they are paths) and also Lemma 3.1 imply the following lemma.

Lemma 3.3 Suppose $\alpha$ is a $\Gamma$-geodesic loop (resp. path) in $N$ and there is a free homotopy (resp. homotopy rel endpoints) which takes $\alpha$ to itself. Then we can deform the homotopy into another free homotopy (resp. homotopy rel endpoints) which fixes $\alpha$ pointwise. 
Suppose $\Gamma \subset N$ is a geodesic link and $j: S \rightarrow N$ is a 2 -incompressible map rel $\Gamma$. For a nonperipheral loop or arc $\alpha \subset S$, we say $\alpha^{*} \subset N$ is the $\Gamma$-geodesic representative of $\alpha$ if $\alpha^{*}$ is the unique $\Gamma$-geodesic that is $\Gamma$-homotopic to $j(\alpha)$. It follows from the definition of 2-incompressible maps that every nonperipheral simple loop $\alpha \subset S$ has a unique $\Gamma$-geodesic representative. Even when $\alpha$ is a nonperipheral simple arc in $S$, it is not hard to see that condition (c) of 2-incompressibility implies that image of $\alpha$ is nonperipheral in $N$ and therefore has a unique $\Gamma$-geodesic representative.

When $S$ is the interior of a finite type surface, by a triangulation of $S$ we mean a finite triangulation where there is a vertex at infinity for every cusp of $S$. More precisely assume $S$ is equipped with a finite area hyperbolic metric, identify the universal cover of $S$ by $\mathbb{H}^{2}$ and consider the triangulation on the compactification of $\mathbb{H}^{2}$ by adding the circle at infinity. Then we consider $\pi_{1}(S)$-equivariant triangulations where the fixed points of the parabolic elements of $\pi_{1}(S)$ are the only ideal points of the triangulation and the projection of the triangulation to $S$ has a finite number of cells.

Definition 3.4 A presimplicial hyperbolic surface in a hyperbolic 3-manifold $N$ is a proper map $f: S \rightarrow N$ of the surface $S$ with a triangulation $T$ such that the restriction of $f$ to each simplex of $T$ is a totally geodesic immersion. With an abuse of notation, we call the image surface a presimplicial hyperbolic surface, and we assume it is equipped with a triangulation which is the image of $T$. A simplicial hyperbolic surface is a presimplicial hyperbolic surface where the cone angle at each vertex is $\geq 2 \pi$.

It is important to point out here that in the presence of a geodesic link $\Gamma$, we only consider presimplicial hyperbolic surfaces that are $\Gamma$-homotopic to a map $j: S \rightarrow$ $N \backslash \Gamma$. As a result, every such map comes with a small deformation that push it off of $\Gamma$. In particular, when we say such a surface is 2 -incompressible or we talk about the $\Gamma$-geodesic representative of a path on such a surface, we automatically assume that we are speaking about this small perturbation.

The following is an immediate consequence of our construction in Lemma 3.2:

Lemma 3.5 (Local view of $\Gamma$-geodesics) If $\alpha$ is a $\Gamma$-geodesic and $x \in \alpha \cap \Gamma$, then either

(i) $\alpha$ is a geodesic segment of $\Gamma$ or

(ii) $\alpha \cap \Gamma$ consists of isolated points and near $x, \alpha$ lies in the union $U$ of two totally geodesic half discs glued along $\Gamma$. With respect to the induced hyperbolic metric on $U, \alpha_{x} \cap U$ is a geodesic where $\alpha_{x}$ is a small neighborhood of $x$ in $\alpha$. 
Using this, the following lemma provides a criterion that guarantees that a presimplicial hyperbolic surface is simplicial hyperbolic. This generalizes an argument of Canary in [6].

Lemma 3.6 (Guaranteeing cone angle $\geq 2 \pi$ ) Let $N$ be a complete hyperbolic 3-manifold and $\Gamma \subset N$ is a geodesic link. Suppose $f: S \rightarrow N$ is a presimplicial hyperbolic surface $\Gamma$-homotopic to a map $j: S \rightarrow N \backslash \Gamma$ and with triangulation $T$. If every vertex of $T$ is on a path $\alpha \subset S$ whose image by $f$ is the $\Gamma$-geodesic representative of $f(\alpha)$ rel endpoints and the $f$-image of the vertex is in the interior of this $\Gamma$-geodesic, then $f$ is a simplicial hyperbolic surface.

Proof Assume $S$ is equipped with the path metric induced by $f$ and $D \subset S$ is a small disc centered at a vertex $v$ of $T$. By assumption, there is a path $\alpha \subset S$ whose $f$-image is a $\Gamma$-geodesic. We assume $D$ is small enough such that the path $\alpha$ divides $D$ into two components; it will be enough to show that the contribution any of these components to the cone angle at $v$ is $\geq \pi$. Let $U$ be the closure of a component of $D \backslash \alpha$ and also assume $x$ and $y$ are the endpoints of the path $\alpha \cap D$. When $D$ is small, the induced metric on $U$ is hyperbolic and therefore if the cone angle of $v$ in $U$ is $\leq \pi$, there is a path $\beta \subset U$ between $x$ and $y$ whose length is smaller than the length of $\alpha \cap D$ and the $f$-image of its interior is disjoint from $\Gamma$. But $f(\beta)$ is $\Gamma$-homotopic to $f(\alpha)$ and has smaller length and this contradicts our assumption that $f(\alpha)$ is the $\Gamma$-geodesic representative of $j(\alpha)$.

Recall that a multiarc is a collection of disjoint, nonperipheral and nonparallel arcs and loops on $S$. Given a multiarc $\gamma \subset S$, we say a simplicial hyperbolic surface $f: S \rightarrow N$ realizes $\gamma$ rel $\Gamma$ if there is a $\Gamma$-homotopy from $j$ to $f$ and the image of (the interior of) $\gamma$ by $f$ is a collection of $\Gamma$-geodesics. Note that if $f$ realizes $\gamma$, the image of a component of $\gamma$ which is an arc, will be a bi-infinite $\Gamma$-geodesic from a cusp of $N$ to a cusp of $N$. The following theorem is essentially due to Soma.

Theorem 3.7 Suppose $\Gamma \subset N$ is a geodesic link in the orientable hyperbolic 3manifold $N$ and $j: S \rightarrow N$ is a 2-incompressible map rel $\Gamma$ of a surface $S$ with $\chi(S)<0$. Then $j$ is $\Gamma$-homotopic to a simplicial hyperbolic surface $f: S \rightarrow N$. Furthermore given a multiarc $\mu, f$ above can be constructed to realize $\mu$ rel $\Gamma$.

Proof We assume $S$ is equipped with a finite area hyperbolic metric and therefore every component of $\partial \bar{S}$ corresponds to a cusp. Without loss of generality assume $\mu=\left\{\alpha_{1}, \ldots, \alpha_{k}\right\}$ is nonempty and every component $\alpha_{i}$ of $\mu$ is given as a closed geodesic or bi-infinite geodesic arc on the chosen hyperbolic metric of $S$. 
By Lemma 3.2 and our comment after the lemma, every $\alpha_{i}, i=1, \ldots, k$, has a unique $\Gamma$-geodesic representative in $N$. After deforming $j$ by a proper $\Gamma$-homotopy, we can obtain a map $f_{0}: S \rightarrow N$ such that $f_{0}\left(\alpha_{i}\right)$ is the $\Gamma$-geodesic representative of $j\left(\alpha_{i}\right)$ and the $f_{0}$-image of $S \backslash \mu$ is disjoint from $\Gamma$. (For the arcs in $\mu$, it might be easier to see this by performing the homotopy in an equivariant way for the lift of $f_{0}$ to the universal covers.) Also since $j$ and therefore $f_{0}$ are proper, one can see that $f_{0}$ (or more precisely its lift to the universal covers) automatically maps every ideal point associated to a cusp of $S$ to an ideal point associated to a cusp of $N$.

Let $V_{0} \subset \mu \cap f_{0}^{-1}(\Gamma)$ consists of those points whose $f_{0}$-images give intersections of $f_{0}\left(\alpha_{i}\right)$ and $\Gamma$ of type (ii) in Lemma 3.5. In particular $V_{0}$ will have finitely many points. Extend $V_{0}$ to a triangulation $T_{0}$ of $S$ such that for every cusp of $S$ there is an ideal vertex of $T_{0}$, all other vertices are on $\mu, V_{0}$ is a subset of the 0 -skeleton of $T_{0}$ and $\mu$ is a subset of the 1 -skeleton of $T_{0}$.

After a $\Gamma$-homotopy, deform $f_{0}$ to a map $f$ which we will construct by starting from $\left.f_{0}\right|_{V_{0}}$ and then extending to the edges and finally extending to the faces of $T_{0}$. Define $f$ on $V_{0}$ in a way that it agrees with $f_{0}$ on the vertices of $T_{0}$ and then extend it to the edges in a way that it takes every edge $e$ of $T_{0}$ to the unique $\Gamma$-geodesic path which is $\Gamma$-homotopic to $f_{0}(e)$ rel endpoints. Note that $f$ will automatically agree with $f_{0}$ on $\mu$. Finally for every triangle $F$ of $T_{0}$, choose a vertex $v$ and the opposite edge $e$. Extend $f$ to $F$ by making the image a ruled triangle consisting of $\Gamma$-geodesics connecting $f(v)$ to $f(x)$ for every $x \in e_{0}$ that are $\Gamma$-homotopic rel endpoints to the arc connecting $f(v)$ to $f(x)$ in the image of the map constructed so far. The continuity of $\Gamma$-geodesic representatives in Lemma 3.2 ensures that this gives a continuous map of $S$ that is $\Gamma$-homotopic to $f_{0}$. We want to show that $f$ is a simplicial hyperbolic surface after refining $T_{0}$ to a triangulation $T$.

Let's consider the preimage of $\Gamma$ in a triangle $F$ of $T$, ie $f^{-1}(\Gamma) \cap F$. By our construction, there is a vertex $v$ of $F$ and the opposite edge $e$ such that $f(F)$ is obtained by coning off $f(e)$ from the point $f(v)$. Using the preimages of these $\Gamma-$ geodesics, we can view $F$ as a union of paths $[v, x]$ where $f([v, x])$ is a $\Gamma$-geodesic connecting $f(v)$ and $f(x)$. The local view of $\Gamma$-geodesics, Lemma 3.5, shows that for every $x \in e$ either $f([x, v])$ is a subarc of a component of $\Gamma$ or it intersects $\Gamma$ finitely many times. For every component $\gamma$ of $\Gamma$, because of the continuity of $f$, the preimage $f^{-1}(\gamma) \cap F$ is closed and will be a finite disjoint union of finite paths that intersects $[v, x]$ finitely many times for every $x \in e$ and also $\operatorname{arcs}\left[v, x_{1}\right], \ldots,\left[v, x_{l}\right]$ for a finite number of points $x_{1}, \ldots, x_{l}$ on $e$. The preimages for different components of $\Gamma$ are disjoint. Hence $f^{-1}(\Gamma) \cap F$ consists of finitely many finite paths. Now triangulate $F$ in a way that the preimage $f^{-1}(\Gamma) \cap F$ is a subset of the one-skeleton. If necessary subdivide further in a way that the union of all these triangulations provides 
a triangulation of $S$ which is a subdivision of $T_{0}$. It is obvious from our construction that the image of the interior of every triangle in the new triangulation $T$ is a ruled triangle disjoint from $\Gamma$. Hence the image of every face of $T$ is totally geodesic and we have shown that $f$ is a presimplicial hyperbolic surface with respect to the triangulation $T$. Even more, in our construction every vertex of $T$ is in the interior of a path $\alpha \subset S$ whose image by $f$ is the interior of the $\Gamma$-geodesic representative of $f(\alpha)$ rel endpoints. The path $\alpha$ is either one of the components of $\mu$ or is a path $[v, x]$ in one of the triangles of $T_{0}$. Therefore and by Lemma 3.6, $f$ is actually a simplicial hyperbolic surface.

The homotopy that is constructed above is not necessarily a $\Gamma$-homotopy and the intermediate surface $f_{0}$ may intersect $\Gamma$ but it should be clear that after a small perturbation, it becomes a $\Gamma$-homotopy and we have proved the theorem.

Definition 3.8 We say a geodesic link $\Gamma$ in $N$ is $\eta$-separated if any path $\alpha: I \rightarrow N$ with endpoints on $\Gamma$ satisfying

$$
l(\alpha(I)) \leq \eta
$$

is homotopic (rel endpoints) into $\Gamma$.

One important property of an $\eta$-separated geodesic link $\Gamma$, which will be used later, is that $\Gamma$ cannot enter a component of the thin parts of $N$ except when it has a component which is the geodesic core of a Margulis tube. We always assume $\eta$ is smaller than the Margulis constant. In this case, more precisely if $T$ is a component of $N \leq \eta / 2$ then $T \cap \Gamma$ is either empty or $T$ is a Margulis tube with the geodesic core $\gamma^{*}$ and $\Gamma \cap T=\gamma^{*}$ is a component of $\Gamma$. In particular $\pi_{1}(T \backslash \Gamma)$ is always abelian.

Lemma 3.9 Suppose $\alpha: S^{1} \rightarrow N$ is a piecewise linear or smooth map to a hyperbolic manifold $N$ which is homotopically trivial in $N$. Then there exists a map $g: D^{2} \rightarrow N$ of the 2-disk $D^{2}$ whose restriction to $S^{1}=\partial D^{2}$ is $\alpha$ and the diameter of $g\left(D^{2}\right)$ in $N$ is bounded from above by the length of $\alpha$ in $N$.

The proof of the above lemma is a simple construction of a ruled disk by taking a point of the curve and use it to cone off all other points. This disk immediately has the required property. Using this we can prove the following.

Lemma 3.10 Suppose $f: S \rightarrow N$ is a simplicial hyperbolic surface which is 2incompressible rel $\Gamma$, an $\eta$-separated geodesic link in the hyperbolic 3-manifold $N$. For every noncontractible simple loop $\alpha$ on $S$ with length $(f(\alpha)) \leq \eta$, the loop $f(\alpha)$ is 
homotopically nontrivial in $N$ and if $\alpha$ is nonperipheral, $f(\alpha)$ is not freely homotopic into a cusp of $N$. In particular

$$
f\left(\Sigma_{f}^{\leq \epsilon}\right) \subset N^{\leq \epsilon}
$$

where $\Sigma_{f}$ denotes the surface $S$ equipped with the path metric induced from $f$ and $\epsilon \leq \eta / 2$.

Proof Suppose a noncontractible simple loop $\alpha$ in $S$ has length $\leq \eta$ in $\Sigma_{f}$ or equivalently the length of $f(\alpha)$ is at most $\eta$. By Lemma 3.9, if $f(\alpha)$ is homotopically trivial in $N$ there exists a map $g: D^{2} \rightarrow N$ where $\left.g\right|_{\partial D^{2}}=\left.f\right|_{\alpha}$ and its diameter is $\leq \eta$. Let $\mu \subset D^{2}$ be an arc with endpoints on $g^{-1}(\Gamma)$ whose $g$-image has length $\leq \eta$. Since $\Gamma$ is $\eta$-separated, $g(\mu)$ is homotopic rel endpoints to an arc $\mu^{\prime}$ of $\Gamma$ whose length also has to be $\leq \eta$. We can perform this homotopy by moving along arcs perpendicular to $\Gamma$ that connect a point of $g(\mu)$ to a point of $\Gamma$. The length of each such arc is $\leq \eta$ and from here, we can see that the homotopy cannot intersect any other point of $\Gamma$. As a result we can isotope $\Gamma$ by pushing $\mu^{\prime}$ off of $g(D)$ and reducing the number intersections of $\Gamma$ and $g(D)$. We can continue such a procedure and we will end up with a link $\Gamma^{\prime}$ isotopic to $\Gamma$ which intersects $g(D)$ at most once. This is however a contradiction to condition (b) in the definition of 2-incompressibility and therefore $f(\alpha)$ could not be homotopically trivial in $N$. Also if $f(\alpha)$ represents a parabolic element of $\pi_{1}(N)$ then $f(\alpha)$ is contained in an $\eta / 2$-thin cusp of $N$ which contradicts condition (c) of 2-incompressibility if $\alpha$ is nonperipheral. The second statement of the lemma follows immediately.

An important property of a simplicial hyperbolic surfaces $f: S \rightarrow N$ and the surface $\Sigma_{f}$ ( $S$ equipped with the induced path metric from $f$ ) is that since the curvature is $\leq-1$ at every point, by the Gauss-Bonnet Theorem, the area of $\Sigma_{f}$ is bounded by $-2 \chi(S)$. Then a standard argument shows that for $\epsilon>0$, every two points $x, y \in N^{\leq \epsilon}$ can be connected by a path that does not intersect $\epsilon$-thin cusps of $\Sigma_{f}$ and whose intersection with $\Sigma_{f}^{\geq \epsilon}$ has length $\leq D(\chi(S), \epsilon)=-2 \pi \chi(S) / \epsilon$. We can use this observation with the previous lemma to show the following:

Lemma 3.11 (Bounded Diameter Lemma) Suppose $\Gamma$ is an $\eta$-separated geodesic link in the hyperbolic 3-manifold $N$ and $f: S \rightarrow N$ is a simplicial hyperbolic surface which is 2 -incompressible rel $\Gamma$. Then for every $0<\epsilon<\eta / 2$,

$$
\operatorname{diam}_{N} \geq \epsilon(f(S)) \leq D(\chi(S), \epsilon) .
$$

Proof By Lemma 3.10 we know

$$
f\left(\Sigma_{f}^{\leq \epsilon}\right) \subset N^{\leq \epsilon}
$$


where $\Sigma_{f}$ is the surface $S$ equipped with the path metric induced from $f$. In addition, we know that the image of a compact component of $\Sigma_{f}^{\leq \epsilon}$ does not map into an $\epsilon$-thin cusp of $N$. As mentioned, every two points $x, y \in \Sigma_{f}^{\geq \epsilon}$ can be connected by a path that avoids $\epsilon$-thin cusps of $\Sigma_{f}$ and the length of its intersection with $\Sigma_{f}^{\geq \epsilon}$ is at most $D(\chi(S), \epsilon)$. The image of this path also avoids $\epsilon-$ thin cusps of $N$ and the total length of its intersection with $N^{\geq \epsilon}$ is bounded by $D(\chi(S), \epsilon)$.

For a simplicial hyperbolic surfaces $f: S \rightarrow N$, we need something stronger than the Bounded Diameter Lemma to tell us that $f$ respects the thick-thin decomposition of $\Sigma_{f}$ and $N$. This is proved in Proposition 3.13. The first issue to resolve is to find uniform thin-thick decompositions for the simplicial hyperbolic surfaces that we use.

Lemma 3.12 Suppose $\Gamma$ is an $\eta$-separated geodesic link in a hyperbolic 3-manifold $N$ and $f: S \rightarrow N$ is a simplicial hyperbolic surface that is 2-incompressible rel $\Gamma$. Also let $\Sigma_{f}$ denotes the surface $S$ equipped with the path metric induced by $f$. Then for any $\epsilon \leq \eta / 8$, every component of $\Sigma_{f}^{\leq \epsilon}$ is either an annular neighborhood of a simple closed geodesic of length $\leq \epsilon$ or is an annular neighborhood of a cusp. Furthermore $\Sigma_{f}^{\geq \epsilon}$ has no annular or disk components.

Proof Recall that the map $f$ comes with a small deformation that pushes it away to a 2-incompressible map in $N \backslash \Gamma$. When speaking of the topological properties or the induced map on the fundamental group, we assume we have already performed such a deformation.

Assume $\epsilon \leq \eta / 8$ and $V$ is a component of $\Sigma_{f}^{\leq \epsilon}$. By Lemma 3.10, the $f$-image of $V$ is contained in a component $T$ of $N \leq \epsilon$. Because $\Gamma$ is $\eta$-separated and by our remark after Definition 3.8, $T \backslash \Gamma$ has an abelian fundamental group.

Suppose $\widetilde{\Sigma}_{f}$ is the universal cover of $\Sigma_{f}$ equipped with the lift of the metric on $\Sigma_{f}$ and $\tilde{V}$ denotes a lift of $V$ to $\widetilde{\Sigma}_{f}$. Given $\tilde{x} \in \tilde{V}$, there exists a primitive element $h_{\tilde{x}} \in \pi_{1}\left(\Sigma_{f}\right)$ such that $d \widetilde{\Sigma}_{f}\left(\tilde{x}, h_{\tilde{x}}(\tilde{x})\right) \leq \epsilon$, where $h_{\tilde{x}}$ acts on $\tilde{\Sigma}_{f}$ as a deck transformation. On the level of fundamental groups, $f$ takes $h_{\tilde{x}}$ to an element of $\pi_{1}(T \backslash \Gamma)$; because $\pi_{1}(T \backslash \Gamma)$ is abelian and $f$ is $\pi$-injective to $N \backslash \Gamma$, we must have $h_{\tilde{x}}=h_{\tilde{y}}$ or $h_{\tilde{x}}=h_{\tilde{y}}^{-1}$ for every $\tilde{x}, \tilde{y} \in \tilde{V}$. (Recall that $h_{\tilde{x}}$ and $h_{\tilde{y}}$ are chosen to be primitive.) This shows that we can choose $h=h_{\tilde{x}}$ independent of $\tilde{x} \in \tilde{V}$ and $\tilde{V}$ is a subset of

$$
\left\{\tilde{x} \in \tilde{\Sigma}_{f}: d_{\tilde{\Sigma}_{f}}(\tilde{x}, h(\tilde{x})) \leq \epsilon\right\} .
$$

Obviously every point in the above set also belongs to $\Sigma_{f}^{\leq \epsilon}$ and because the function $d(\cdot, h(\cdot))$ is convex, the above set is connected and convex. As a result it must be equal to $\widetilde{V}$. 
Next we claim that $V$, the projection of $\tilde{V}$ to $\Sigma_{f}$, is the same as $\tilde{V} /\langle h\rangle$, the quotient of $\widetilde{V}$ by the action of $h$, and therefore is a noncontractible annulus. This follows if we show that for every $g \in \pi_{1}\left(\Sigma_{f}\right), g \widetilde{V} \cap \tilde{V}=\varnothing$ unless $g$ is a power of $h$. If $g \tilde{V} \cap \tilde{V} \neq \varnothing$ then because of the convexity of $\tilde{V}$, we can see that the induced map, on the fundamental groups, takes $g$ to an element of $\pi_{1}(T \backslash \Gamma)$. Again by using the fact that $\pi_{1}(T \backslash \Gamma)$ is abelian and $f$ is $\pi_{1}$-injective to $N \backslash \Gamma$, it follows that $g$ must be a power of $h$. This proves the claim and as a corollary, we see that $V$ is an essential annulus in $\Sigma_{f}$.

So obviously $\Sigma_{f}^{\geq \epsilon}$ has no disk components. Two parallel annuli cannot be components of $\Sigma_{f}^{\leq \epsilon}$ because then it means that we can lift them to disjoint sets $\tilde{V}_{1}, \widetilde{V}_{2}$ in $\widetilde{\Sigma}_{f}$ and find a primitive element $h \in \pi_{1}\left(\Sigma_{f}\right)$ such that

$$
V_{1}=\tilde{V}_{1} /\langle h\rangle \text { and } V_{2}=\tilde{V}_{2} /\langle h\rangle
$$

But then our characterization above shows that we must have

$$
\widetilde{V}_{1}=\widetilde{V}_{2}=\left\{\tilde{x} \in \widetilde{\Sigma}_{f}: d_{\widetilde{\Sigma}_{f}}(\tilde{x}, h(\tilde{x})) \leq \epsilon\right\},
$$

and $V_{1}=V_{2}$ could not be disjoint.

An annular neighborhood of every cusp of $\Sigma_{f}$ is contained in $\Sigma_{f}^{\leq \epsilon}$ so the above argument repeated when $h$ is a parabolic element of $\pi_{1}\left(\Sigma_{f}\right)$ shows that $\Sigma_{f}^{\leq \epsilon}$ does not contain a component which is a boundary parallel compact annulus. These two facts together show that $\Sigma_{f}^{\geq \epsilon}$ has no annular component either.

Now using our observations above, we can show that in some sense $f$ respects the thick-thin decomposition.

Proposition 3.13 (Thick goes to thick) Given $\eta>0$ smaller than the Margulis constant and surface $S$ there exists a continuous map $\rho_{0}:[0, \infty) \rightarrow[0, \infty)$ with $\rho_{0}(\epsilon) \leq \epsilon$ for every $\epsilon \geq 0$ where the following holds. Suppose $\Gamma$ is an $\eta$-separated geodesic link in the hyperbolic 3-manifold $N$ and $f: S \rightarrow N$ is a simplicial hyperbolic surface, which is in addition 2-incompressible rel $\Gamma$. Then for every $\epsilon \leq \eta / 2$

$$
f\left(\Sigma_{f}^{\leq \epsilon}\right) \subset N^{\leq \epsilon} \quad \text { and } \quad f\left(\Sigma_{f}^{\geq \epsilon}\right) \subset N^{\geq \rho_{0}(\epsilon)},
$$

where $\Sigma_{f}$ denotes the surface $S$ equipped with the path metric induced from the $\operatorname{map} f$.

Proof The first inclusion is what we have already proved in Lemma 3.10. Also note that our explanation before the Bounded Diameter Lemma 3.11 shows that diameter of every component of $\Sigma_{f}^{\geq \epsilon}$ is at most $D(\chi(S), \epsilon)=-2 \pi \chi(S) / \epsilon$. Assume $\epsilon<\eta / 8$; 
then Lemma 3.12 shows the fundamental group of each component of $\Sigma_{f}^{\geq \epsilon}$ contains a free group of rank 2 .

Choose $\delta \leq \epsilon$ to be small enough so that $\xi(\eta / \delta) \geq D(\chi(S), \epsilon)$, where $\xi(\cdot)$ is the function obtained in Lemma 2.2, and suppose the image of a point $x \in \Sigma_{f}^{\geq \epsilon}$ is contained in $N^{<\delta}$. By Lemma 2.2, the image of the component $R$ of $\Sigma_{f}^{\geq \epsilon}$ which contains $x$, is contained in a component $T$ of $N \leq \eta$. Similar to the proof of the above lemma, $\pi_{1}(T \backslash \Gamma)$ is abelian and after a small deformation we can assume the image of $R$ is contained in $T \backslash \Gamma$ but this contradicts $\pi$-injectivity of the map to $N \backslash \Gamma$ and the fact that $\pi_{1}(R)$ contains a free group of rank 2. Hence $f(x) \in N^{\geq \rho_{0}(\epsilon)}$ where

$$
\rho_{0}(\epsilon)=\min \left(\epsilon, \eta / \xi^{-1}\left(\frac{-2 \pi \chi(S)}{\epsilon}\right)\right) .
$$

When $\epsilon>\eta / 8$ then obviously $\Sigma_{f}^{\geq \epsilon} \subset \Sigma_{f}^{\geq \eta / 8}$ and we can simply define

$$
\rho_{0}(\epsilon)=\rho_{0}(\eta / 8) \text {. }
$$

An immediate corollary of the above proposition is the existence of uniformly wide collar neighborhoods of short geodesics on $\Sigma_{f}$ for a map $f$ that satisfies the hypothesis. We should point out that in case the induced metric on $\Sigma_{f}$ is hyperbolic, then the existence of such collar neighborhoods is a consequence of the Collar Lemma for hyperbolic surfaces. However for simplicial hyperbolic surfaces and the induced singular hyperbolic metrics, in general, it is impossible to expect uniformly wide collar neighborhoods. We find such collar neighborhoods below but the width will depend on $\eta$ as well as on $\chi(S)$.

Corollary 3.14 With the same hypothesis as of Proposition 3.13, assume $\epsilon_{1}, \epsilon_{2}>0$ are given and both are smaller than $\eta / 2$. Then the distance between $\Sigma_{f}^{\geq \epsilon_{1}}$ and $\Sigma_{f}^{\leq \epsilon_{2}}$ is at least $\xi\left(\rho_{0}\left(\epsilon_{1}\right) / \epsilon_{2}\right)$ where $\rho_{0}$ and $\xi$ are the functions described in Proposition 3.13 and Lemma 2.2 and $\epsilon_{2}<\rho_{0}\left(\epsilon_{1}\right)$. In particular, if $\gamma$ is an essential loop of length at most $\epsilon<\rho_{0}(\eta / 2)$ on $\Sigma_{f}$, the distance between $\gamma$ and the boundary of the associated component of $\Sigma_{f}^{\leq \eta / 2}$ containing $\gamma$ is at least $\xi\left(\rho_{0}(\eta / 2) / \epsilon\right)$.

\section{Quasi-convexity}

Suppose $N$ is a hyperbolic 3-manifold and $\Gamma$ is an $\eta$-separated geodesic link for some $\eta>0$. Also assume $S$ is the interior of a surface of finite type with $\chi(S)<0$ and $j: S \rightarrow N$ is a map that is 2 -incompressible rel $\Gamma$. 
Definition 4.1 Given $B>0$, we define $\mathcal{C}(B, j) \subset \mathcal{C}_{0}(S)$ to be the set of homotopy classes of simple loops $\alpha \in \mathcal{C}_{0}(S)$ whose $\Gamma$-geodesic representative in $N$ has length at most $B$.

Recall an elementary observation of Bers which shows that for every surface of finite type $S$, there exists a constant $B_{0}(S)$, which we call the Bers' constant, such that for every finite area hyperbolic metric on $S$, there is a nonperipheral essential simple loop whose length is at most $B_{0}(S)$. It is easy to see that the same argument and the same constant $B_{0}(S)$ works for singular finite area hyperbolic metrics with cone angles at least $2 \pi$.

Our main goal in this section is to show that for $B$ larger than the Bers' constant, $\mathcal{C}(B, j)$ is quasi-convex with a quasi-convexity constant which depends only on $\chi(S), \eta$ and $B$.

Remark 4.2 Note that in the above definition, we have ignored elements of $\mathcal{C}_{0}(S)$ which are represented by simple arcs in $\bar{S}$. But they will be used in the proof of Lemma 4.4 and, especially in the case of once holed torus or four holed sphere, we use these arcs since distinct loops of $\mathcal{C}_{0}(S)$ intersect.

Given a simplicial hyperbolic surface $f: S \rightarrow N$ and $B>0$, by $\operatorname{short}(f, B)$ we denote the set of loops in $\mathcal{C}_{0}(S)$ whose geodesic representatives in the metric induced by $f$ has length at most $B$. Using this, we define a projection $\Pi_{j, B}(\alpha)$ for every $\alpha \in \mathcal{C}_{0}(S)$ as follows:

$$
\Pi_{j, B}(\alpha)=\bigcup_{f} \operatorname{short}(f, B),
$$

where the union is over all simplicial hyperbolic surfaces which are $\Gamma$-homotopic to $j$ and realize $\alpha$. Note that by Theorem 3.7, the set of such maps is nonempty. Also note that in our definition, we allow $\alpha$ to be the homotopy class of an arc.

In order to prove the quasi-convexity of $\mathcal{C}(B, j)$, we will prove in Proposition 4.5 that the above projection is a coarse projection and then this immediately implies the quasi-convexity. This will essentially boil down to the following two lemmas which show that the projection is well defined as a coarse map. Our first result is to show that $\operatorname{short}(f, B)$ has bounded diameter in $\mathcal{C}(S)$ for a single element $f$ used in the above union.

Lemma 4.3 Suppose $f: S \rightarrow N$ is a simplicial hyperbolic surface which is 2incompressible rel $\Gamma$, an $\eta$-separated geodesic link in $N$. For every $\alpha, \beta \in \operatorname{short}(f, B)$, $i(\alpha, \beta)$ is bounded by a constant that depends only on $\chi(S), \eta$ and $B$. As a result, $\operatorname{diam}_{\mathcal{C}}(\operatorname{short}(f, B))$ is bounded by a constant that depends only on $\chi(S), \eta$ and $B$. 
Proof The first part is a consequence of Corollary 3.14. Assume $\Sigma_{f}$ is the surface $S$ equipped with the singular hyperbolic metric induced from the map $f$. We consider all the lengths and distances in this metric and assume $\alpha$ and $\beta$ are geodesic closed curves. Consider $\epsilon>0$ very small in a way that will be determined in what follows. Also suppose

$$
i(\alpha, \beta)>\lceil B / \epsilon\rceil^{2}
$$

where $\lceil x\rceil$ denotes the smallest integer that is greater than or equal to $x$. We can divide $\alpha$ into $\lceil B / \epsilon\rceil$ segments of length at most $\epsilon$ and by the Pigeonhole Principle, there will be at least $\lceil B / \epsilon\rceil+1$ points of $\alpha \cap \beta$ in one of these segments. Similarly we can divide $\beta$ into $\lceil B / \epsilon\rceil$ segments of length at most $\epsilon$ and among the points of $\alpha \cap \beta$ which we have already chosen there will be two points $p, q$ in one of these segments. This way we find $p$ and $q$ in $\alpha \cap \beta$ with an arc $\kappa_{\alpha} \subset \alpha$ and an $\operatorname{arc} \kappa_{\beta} \subset \beta$ both with lengths at most $\epsilon$ and endpoints $p$ and $q$. The concatenation of these arcs gives a closed curve $\gamma=\kappa_{1} * \kappa_{2}$ of length at most $2 \epsilon$. This is an essential closed curve on $S$ and by Corollary 3.14, it is contained in an annular component of $\Sigma_{f}^{\leq \eta / 2}$ and the distance from $\gamma$ to the boundary components of this annulus is at least $\xi\left(\rho_{0}(\eta / 2) /(2 \epsilon)\right)$. So if we choose $\epsilon$ small enough such that

$$
\xi\left(\frac{\rho_{0}(\eta / 2)}{2 \epsilon}\right)>B
$$

then $\alpha$ and $\beta$ both have to be contained in this annulus. But $\alpha$ and $\beta$ are simple and must be freely homotopic to the core of the annulus which then implies $i(\alpha, \beta)=0$ which is a contradiction and we must have $i(\alpha, \beta) \leq\lceil B / \epsilon\rceil^{2}$.

The second statement follows from an elementary argument (see Masur and Minsky [11]) that shows $d_{\mathcal{C}}(\alpha, \beta) \leq 2 i(\alpha, \beta)+1$.

The next lemma shows that if $f$ and $g$ are two simplicial hyperbolic surfaces used in the definition of $\Pi_{j, B}(\alpha)$ then $\operatorname{short}(f, B)$ and $\operatorname{short}(g, B)$ intersect for $B$ sufficiently large. Together with the previous lemma, it proves that $\Pi_{j, B}(\alpha)$ has bounded diameter (depending on $B, \chi(S)$ and $\eta)$ in $\mathcal{C}(S)$ for every $B$. As before, we denote the surface $S$ equipped with the metrics induced by $f$ and $g$ by $\Sigma_{f}$ and $\Sigma_{g}$ respectively.

Lemma 4.4 Suppose $j: S \rightarrow N$ is 2-incompressible rel $\Gamma$, an $\eta$-separated geodesic link in the hyperbolic 3-manifold $N$, and $\eta>0$ is smaller than the Margulis constant. If $f, g: S \rightarrow N$ are simplicial hyperbolic, both are $\Gamma$-homotopic to $j$ and both realize a nonperipheral simple loop or arc $\alpha$, there exists a nonperipheral simple loop $\gamma$ whose lengths in $\Sigma_{f}$ and $\Sigma_{g}$ are bounded by a constant that depends only on $\chi(S)$ and $\eta$. 
Proof By Proposition 3.13,

$$
f\left(\Sigma_{f}^{\geq \epsilon}\right) \subset N^{\geq \rho_{0}(\epsilon)}
$$

for every $\epsilon \in(0, \eta / 2]$. Assume $\alpha$ represents the geodesic representative of $\alpha$ in $\Sigma_{f}$ and $\Sigma_{g}$. Since $f$ and $g$ take these geodesic representatives to the unique $\Gamma$-geodesic representative of $\alpha$ in $N$, we use $f$ and $g$ to identify copies of $\alpha$ on $\Sigma_{f}$ and $\Sigma_{g}$. In addition, whenever necessary, we assume $f$ and $g$ have been perturbed slightly in a way that $f(S), g(S) \subset N \backslash \Gamma$. (Compare with our remark after Definition 3.4.)

Recall that there exists a constant $\epsilon_{0}$ such that in every hyperbolic surface $\Sigma$, whenever a simple geodesic enters a component of $\Sigma^{\leq \epsilon_{0}}$, it has to crosses that component, ie it has to cross every closed curve homotopic to the core of that component. Assume $\alpha$ enters a compact component of the $\delta$-thin part of $\Sigma_{f}$ where $\delta=\min \left\{\rho_{0}(\eta / 8), \epsilon_{0}\right\}$ and assume $p$ is a point of $\alpha$ in such a component. Then by Proposition 3.13, $f(p) \in N \leq \delta$ and since $f(p)=g(p)$, using Proposition 3.13 once more, we have $p \in \Sigma_{g}^{\leq \eta / 8}$. As a result, there exist noncontractible simple loops $\gamma_{f}, \gamma_{g}$ based at $p$ where the length of $\gamma_{f}$ in $\Sigma_{f}$ and the length of $\gamma_{g}$ in $\Sigma_{g}$ do not exceed $\eta / 8$. Let $T$ be the component of $N^{\leq \eta / 8}$ which contains $f(p)=g(p)$. We obviously have $f\left(\gamma_{f}\right), g\left(\gamma_{g}\right) \subset T$ and since $f$ and $g$ are homotopic in $N \backslash \Gamma$, the loop $f\left(\gamma_{g}\right)$ (as a loop based at $f(p)$ ) is also homotopic in $N \backslash \Gamma$ to a loop contained in $T \backslash \Gamma$ and therefore $f\left(\gamma_{f}\right)$ and $f\left(\gamma_{g}\right)$ represent two elements of $\pi_{1}(T \backslash \Gamma)$. Recall from our remark after Definition 3.8 that $\pi_{1}(T \backslash \Gamma)$ is abelian; hence $f\left(\gamma_{f}\right)$ and $f\left(\gamma_{g}\right)$ commute as elements of $\pi_{1}(T \backslash \Gamma)$. But $f$ is $\pi_{1}$-injective as a map to $N \backslash \Gamma$ and since $\gamma_{f}$ and $\gamma_{g}$ are primitive, we must have that they are homotopic (as unoriented loops) and in particular $\gamma_{g}$ is nonperipheral. This provides a simple nonperipheral loop whose length in $\Sigma_{f}$ and $\Sigma_{g}$ is at most $\eta / 2$ and we have proved the conclusion in this case.

Hence we can assume $\alpha$ does not enter any compact component of $\Sigma_{f}^{\leq \delta}$ and using a similar argument we also assume it does not enter any compact component of $\Sigma_{g}^{\leq \delta}$. Note that by our choice $\delta \leq \epsilon_{0}$, it implies that if $\alpha$ is a loop then it stays completely in $\Sigma_{f}^{\geq \delta}$ and $\Sigma_{g}^{\geq \delta}$. Even when $\alpha$ is an arc, it has an initial and terminal subsegments that exit and enter a cusp but remains in the $\delta$-thick parts otherwise.

Suppose $\kappa:[0, \ell] \rightarrow \alpha$ parametrizes $\alpha \cap \Sigma_{f}^{\geq \delta}$ with respect to the arc length, where $\ell$ is the length of $\alpha \cap \Sigma_{f}^{\geq \delta}$ and as mentioned before we are viewing $\alpha$ as a geodesic in $\Sigma_{f}, \Sigma_{g}$ and $N$ simultaneously. Again using Proposition 3.13, we can see that image of $\kappa$ has to be in $N^{\geq \rho_{0}(\delta)}$ and in $\Sigma_{g}^{\geq \rho_{0}(\delta)}$.

Let $v=\rho_{0}(\delta) / 4$ and $K=1+4[\chi(S)]^{2} / v^{4}$. If $\ell<K$, either $\alpha$ itself is a simple non-peripheral loop whose length in both $\Sigma_{f}$ and $\Sigma_{g}$ is bounded by $K$, or using the image of $\kappa$ and surgery with the boundary components of $\Sigma_{f}^{\geq \delta}$ we can produce 
a simple nonperipheral loop whose length in $\Sigma_{f}$ and $\Sigma_{g}$ is at most $K+2 \rho_{0}^{-1}(\delta)$. (Using a similar argument to the above, it is not hard to see that $\kappa(0)$ and $\kappa(\ell)$ have to be in the $\rho_{0}^{-1}(\delta)$-thin part of $\Sigma_{g}$.)

Suppose $\ell \geq K$; in this case, we claim there exist $0 \leq a<b \leq K$ such that $\kappa(a)$ and $\kappa(b)$ are closer than $2 v$ both in $\Sigma_{f}$ and $\Sigma_{g}$ but the subarc of $\alpha$ connecting them has length $\geq 1$. To prove the claim, let $M=\Sigma_{f} \times \Sigma_{g}$ be the product 4-manifold with the product metric. The curvature of $\Sigma_{f}$ and $\Sigma_{g}$ at all points is $\leq 1$ and therefore by Gauss-Bonnet, the area of each of them is at most $-2 \pi \chi(S)$. By Fubini's Theorem, the volume of $M$ is the product of these areas and is at most $4 \pi^{2}[\chi(S)]^{2}$. Because $\alpha \subset \Sigma_{\bar{f}}^{\geq \delta}$ and $\alpha \subset \Sigma_{g}^{\geq \rho_{0}(\delta)}$ and $\nu<\rho_{0}(\delta) \leq \delta$, for every point $(\kappa(t), \kappa(t)) \in M$ the product

$$
D_{M}^{v}(\kappa(t), \kappa(t))=B_{\Sigma_{f}}(\kappa(t), v) \times B_{\Sigma_{g}}(\kappa(t), v)
$$

is an embedded product of disks in $M$, where $B_{\Sigma}(x, r)$ denotes the open disk of radius $r$ centered at $x$ in $\Sigma$. Again using the upper bound for the curvature, the areas of $B_{\Sigma_{f}}(\kappa(t), v)$ and $B_{\Sigma_{g}}(\kappa(t), v)$ are at least $\pi v^{2}$ and therefore the volume of $D_{M}^{v}(\kappa(t), \kappa(t))$ is at least $\pi^{2} v^{4}$.

If $\ell \geq K$ then, because of the upper bound for the volume of $M$, the sets

$$
D_{M}^{v}(\kappa(n), \kappa(n)), \quad n \in \mathbb{Z} \cap[0, K-1]
$$

cannot be all disjoint and therefore we find $0 \leq a<b \leq K$ with

$$
D_{M}^{v}(\kappa(a), \kappa(a)) \cap D_{M}^{v}(\kappa(b), \kappa(b)) \neq \varnothing
$$

and such that the subarc of $\alpha$ connecting $\kappa(a)$ and $\kappa(b)$ has length $\geq 1$. This immediately implies that the distance between $\kappa(a)$ and $\kappa(b)$ in both $\Sigma_{f}$ and $\Sigma_{g}$ is strictly less than $2 v=\rho_{0}(\delta) / 2$. Choose an $\operatorname{arc} \mu_{f} \subset \Sigma_{f}$ and an arc $\mu_{g} \subset \Sigma_{g}$ of length $<2 v$ with endpoints $\kappa(a)$ and $\kappa(b)$. By concatenating these arcs with $\kappa[a, b]$, we obtain loops

$$
\gamma_{f}=\kappa[a, b] * \mu_{f} \subset \Sigma_{f} \quad \text { and } \quad \gamma_{g}=\kappa[a, b] * \mu_{g} \subset \Sigma_{g}
$$

whose lengths are bounded by $K+2 v$. We claim these two loops represent freely homotopic loops in $S$. Because $f$ and $g$ are $\pi_{1}$-injective into $N \backslash \Gamma$, it will be enough to show $f\left(\mu_{f}\right)$ and $g\left(\mu_{g}\right)$ are homotopic (rel endpoints) in $N \backslash \Gamma$.

The closed curve $\omega=f\left(\mu_{f}\right) * g\left(\mu_{g}\right)$ has length strictly less than $4 v=\rho_{0}(\delta)$. We know $\kappa(a) \in \Sigma_{f}^{\geq \delta}$ and by Proposition 3.13, $f(\kappa(a)) \in N^{\geq \rho_{0}}(\delta)$. Hence every loop of length $<\rho_{0}(\delta)$ based at $f(\kappa(a))$ has to be homotopically trivial in $N$. By Lemma 3.9 and since $\Gamma$ is $\eta$-separated, either $\omega$ is homotopically trivial in $N \backslash \Gamma$ or $\omega$ is a multiplied meridian of a component of $\Gamma$ in $N$. We claim there is an $\operatorname{arc} \mu_{g}^{\prime} \subset \Sigma_{f}$ whose image by $f$ is homotopic rel endpoints to $g\left(\mu_{g}\right)$ in $N \backslash \Gamma$. This simply follows 
from Lemma 3.3 and the observation that in the homotopy relating $f$ and $g$, we can assume $\alpha$ remains fixed pointwise. Hence $f\left(\mu_{g}^{\prime} * \mu_{f}\right)$ is homotopic in $N \backslash \Gamma$ to $\omega$ and therefore is either homotopically trivial in $N \backslash \Gamma$ or is a multiplied meridian of a component of $\Gamma$. Yet $f$ is 2 -incompressible and therefore $f\left(\mu_{g}^{\prime} * \mu_{f}\right)$ and as a result $\omega$ must be homotopically trivial in $N \backslash \Gamma$. This proves that $f\left(\mu_{f}\right)$ and $g\left(\mu_{g}\right)$ are homotopic (rel endpoints) in $N \backslash \Gamma$ and we have proved the lemma.

Finally using the above two lemmas, we can prove the main properties of the projection $\Pi_{j, B}$.

Proposition 4.5 Suppose $j: S \rightarrow N$ is 2-incompressible rel $\Gamma$, an $\eta$-separated geodesic link in the hyperbolic 3-manifold $N$ and $B \geq B_{0}(S)$ is given. There exists a constant $c_{B}$ depending only on $\chi(S), \eta$ and $B$ such that the following holds:

- (Coarse idempotence) If $\alpha \in \mathcal{C}(B, j)$ then $\alpha \in \Pi_{j, B}(\alpha)$.

- (Coarse Lipschitz) For $\alpha$ and $\beta \in \mathcal{C}_{0}(S)$ with $d_{\mathcal{C}}(\alpha, \beta) \leq 1$,

$$
\operatorname{diam}_{\mathcal{C}}\left(\Pi_{j, B}(\alpha) \cup \Pi_{j, B}(\beta)\right) \leq c_{B} .
$$

Proof The proof of (a) follows immediately from Theorem 3.7.

For part (b), note that $\alpha$ and $\beta$ are disjoint and therefore $\alpha \cup \beta$ is a multiarc on $S$. Again by applying Theorem 3.7, one knows there exists a simplicial hyperbolic surface $f$ that is $\Gamma$-homotopic to $j$ and realizes $\alpha \cup \beta$. Let $\bar{B}$ be the larger of $B$ and the constant obtained in Lemma 4.4. Then we know that if $g$ is a simplicial hyperbolic surface, used in the definition $\Pi_{j, B}(\alpha)$ or $\Pi_{j, B}(\beta)$, then $\operatorname{short}(f, \bar{B}) \cap \operatorname{short}(g, \bar{B})$ is nonempty. Obviously $\operatorname{short}(g, B) \subset \operatorname{short}(g, \bar{B})$ for every such $g$ and by Lemma 4.3, the diameter of $\operatorname{short}(g, \bar{B})$ is bounded depending on $\chi(S), \eta$ and $B$. This implies part (b) of the proposition.

Recall that $\mathcal{C}(B, j)$ denotes the set of simple closed curves on $S$ whose $\Gamma$-geodesic representatives have length $\leq B$. Once we have the above properties of the projection, we can use the following observation of Minsky [16, Lemma 3.3].

Lemma 4.6 Let $X$ be a $\delta$-hyperbolic geodesic metric space and $Y \subset X$ a subset admitting a map $\Pi: X \rightarrow Y$ which is coarse-Lipschitz and coarse idempotent. That is, there exists $C>0$ such that

- if $d(x, y) \leq 1$ then $d(\Pi(x), \Pi(y)) \leq C$, and

- if $y \in Y$ then $d(y, \Pi(y)) \leq C$. 
Then $Y$ is $K$-quasi-convex for some $K=K(\delta, C)$, and furthermore if $g$ is a geodesic in $X$ whose endpoints are within distance $a$ of $Y$ then

$$
d(x, \Pi(x)) \leq b
$$

for some $b=b(a, \delta, C)$.

We use this to prove the quasi-convexity of $\mathcal{C}(B, j)$ which obviously implies Theorem 1.1.

Theorem 4.7 (Quasi-convexity theorem) Suppose $j: S \rightarrow N$ is 2-incompressible rel $\Gamma$, an $\eta$-separated geodesic link in $N$. Given $B \geq B_{0}(S)$, there exists $L$ depending only on $\chi(S), \eta$ and $B$ such that the $\operatorname{set} \mathcal{C}(B, j)$ is $L$-quasi-convex in $\mathcal{C}(S)$. Moreover if $\beta$ is a geodesic in $\mathcal{C}(S)$ with endpoints in $\mathcal{C}(B, j)$ then $d_{\mathcal{C}}\left(x, \Pi_{j, B}(x)\right) \leq L$ for each $x \in \beta$.

\section{Finding geodesic links}

In order to prove Theorem 1.2 in the most general case, we need to consider pared 3manifolds. Suppose $M$ is a compact, oriented, irreducible, 3-manifold with nonempty boundary and $M$ is neither a 3-ball nor a solid torus. In addition assume $P \subset \partial M$ is a compact subsurface such that, every component of $P$ is an incompressible annulus or a torus and every map $c:\left(S^{1} \times I, S^{1} \times \partial I\right) \rightarrow(M, P)$ which induces an injection on the fundamental groups, is homotopic, as a map of pairs, to a map whose image is contained in $P$. Here we will deal only with pared 3-manifolds without torus boundary components and in this case we require $\pi_{1}(M)$ not to have any noncyclic abelian subgroups. In general, one requires the noncyclic abelian subgroups to be conjugate to a subgroup of the fundamental group of a component of $P$. We say $(M, P)$ is a pared $3-$ manifold. Also with an abuse of notation, we say a loop in $M$ is nonperipheral if it is noncontractible and cannot be homotoped into a component of $P$.

When $(M, P)$ is a pared 3-manifold, every component of $\partial M \backslash P$ is called a free side. By a proper embedding of $(M, P)$ into a hyperbolic 3-manifold $N$, we mean a proper embedding $j: M \backslash P \rightarrow N$ such that the $j$-image of $\mathcal{N}(P) \backslash P$ is contained in the union of the rank one cusps of $N$ where $\mathcal{N}(P)$ is a regular neighborhood of $P$. If $E$ is a free side of $(M, P)$, we say it is incompressible to the outside if given an embedding of a disk $\iota: D^{2} \hookrightarrow N$ with $\iota(D) \cap j(M)=\iota\left(\partial D^{2}\right) \subset j(E)$, it is possible to homotope the map through maps that take $\partial D$ to $j(E)$ into a map whose image is contained in $j(E)$.

We also say a proper embedding $j: M \backslash P \rightarrow N$ is 2-incompressible rel a geodesic link $\Gamma \subset N$ if 
(a) $j(M \backslash P) \cap \Gamma=\varnothing$, and $j_{*}: \pi_{1}(M) \rightarrow \pi_{1}(N \backslash \Gamma)$ is injective,

(b) the $j$-image of a noncontractible loop in $M$ is not freely homotopic in $N \backslash \Gamma$ to a (multiplied) meridian of any component of $\Gamma$ and

(c) given a neighborhood of $U$ of a cusp of $N$ with $U \cap \Gamma=\varnothing$, the $j$-image of a simple loop on a free side of $(M, P)$, which is nonperipheral in $(M, P)$, cannot be homotoped into $U$.

As mentioned in the introduction for the case when $S$ is closed and $N$ has no parabolics, a proper embedding $S \rightarrow N$ of the interior of an oriented surface of finite type $\bar{S}$ is 2 -incompressible rel $\Gamma$ if and only if a collared neighborhood of its image, as a proper image of the pared manifold $(\bar{S} \times I, \partial \bar{S} \times I)$, is 2-incompressible rel $\Gamma$.

Let $E$ be the interior of an oriented surface of finite type and with negative Euler characteristic. Also let $W$ be a complete hyperbolic 3-manifold homeomorphic to $E \times \mathbb{R}$. Recall that $W$ is doubly degenerate if it is identical to its convex core. It follows from work of Canary [6] that in this situation, one can sweep out through $W$ by simplicial hyperbolic surfaces, ie there exists a continuous map $E \times \mathbb{R} \rightarrow W$ which is onto, induces a homotopy equivalence and the image of $E \times\{t\}$ is a simplicial hyperbolic surface for every $t \in \mathbb{R}$. The large scale geometry of such hyperbolic 3 -manifolds is understood by works of Minsky [15] and Brock, Canary and Minsky [2] and in particular there are bilipschitz models of these 3-manifolds which are constructed by using the combinatorial information provided by the complex of curves of $E$ and work of Masur and Minsky [11; 12].

Suppose $N$ is a hyperbolic 3-manifold and the surface $E$ is fixed; by an $L$-product region in $N$, we mean a submanifold $U \subset N$ homeomorphic to $E \times[-1,1]$ such that there is a doubly degenerate hyperbolic 3-manifold $W$ homeomorphic to $E \times \mathbb{R}$ and a homotopy equivalence $\phi: U \rightarrow W$ with the following properties:

- $\phi$ is an $L$-bilipschitz embedding.

- There are simplicial hyperbolic surfaces $X_{-}, X_{+} \subset W$ such that $\phi(E \times\{ \pm 1\}) \subset$ $\mathcal{N}\left(X_{ \pm}\right)$.

As before we let $U_{\epsilon}=U \cap N_{\epsilon}$ denote the subset of $U$ obtained by removing the interiors of all $\epsilon$-cusps. We define the $\epsilon$-width of $U$ to be the distance in $U_{\epsilon}$ between boundary components of $U$ rel $N \leq \epsilon$, ie the minimum length of the intersection with $N^{\geq \epsilon}$ of an arc in $U_{\epsilon}$ that connects the two boundaries of $U$. A level surface $\Sigma \subset U$ is the image of a proper embedding $E \hookrightarrow U$ which is a homotopy equivalence.

The goal of this section is to show that given a proper embedding of a pared 3-manifold into a hyperbolic $3-$ manifold, large product regions can be used to find $\eta$-separated 
geodesic links which make the embedding 2-incompressible. Even more the total length of the obtained geodesic link will be bounded depending on $\chi(E)$ and $\eta$ will be bounded below by a universal constant. First we need to show that in a wide product region we can find lots of closed geodesic curves with definite tube radii and large pairwise distances.

Using our comment before the Bounded Diameter Lemma 3.11, we know there is a bound $D(\chi(E), \epsilon)$ for the diameter of every simplicial hyperbolic surface relative to the $\epsilon$-thin parts. Now we claim the following proposition. Recall from before that $B_{0}(E)$, the Bers' constant, is a constant depending only on $\chi(E)$ such that on every finite area hyperbolic metric on $E$, there exists a nonperipheral noncontractible simple loop of length $\leq B_{0}(E)$.

Proposition 5.1 Given $n, L$ and $\epsilon_{0}$ if $\omega$ is sufficiently large, in an $L$-product region $U \subset N$ of $\epsilon_{0}$-width $\omega$ there exists a collection $\gamma_{1}, \gamma_{2}, \ldots, \gamma_{n}$ of closed geodesics and an embedding $\psi: E \times[0, n] \rightarrow U$ such that

- $\psi$ induces a homotopy equivalence,

- each $\gamma_{i}$ has tube radius $\geq 0.025$ and length $\leq L B_{0}(E), i=1, \ldots, n$,

- $\gamma_{i} \subset \psi(E \times(i-1, i)), i=1, \ldots, n$,

- $\operatorname{diam}_{N}^{\geq \epsilon_{0}}(\psi(E \times\{i\}))$ is bounded by a constant depending only on $L, \epsilon_{0}$ and $\chi(E)$,

- $d_{N}^{\geq \epsilon_{0}}(\psi(E \times\{i-1\}), \psi(E \times\{i\})) \geq D\left(\chi(S), \epsilon_{0}\right), i=1, \ldots, n$,

- $d_{N}^{\geq \epsilon_{0}}\left(\gamma_{i}, \psi(E \times\{i-1\})\right)$ and $d_{N}^{\geq \epsilon_{0}}\left(\gamma_{i}, \psi(E \times\{i\})\right)$ are at least $1, i=1, \ldots, n$.

Recall that in our definition of $d_{N}^{\geq \epsilon_{0}}$ and $\operatorname{diam}_{N}^{\geq \epsilon_{0}}$ in Section 2, we only considered paths that avoid $\epsilon_{0}$-thin cusps of $N$.

Proof By definition there is a doubly degenerate hyperbolic 3-manifold $W$ and a homotopy equivalence $\phi: U \rightarrow W$ which is an $L$-bilipschitz embedding. Let $V=\phi(U)$; then obviously $V$ is a 1-product region and it makes sense to speak of the $\epsilon$-width of $V$ for any $\epsilon>0$. When $\epsilon_{0}$-width of $U$ is at least $\omega$ then the $\epsilon_{1}$-width of $V$ is at least $\omega / L$ for $\epsilon_{1}=\epsilon_{0} / L$. We know that every point of $W$ belongs to a simplicial hyperbolic surface homeomorphic to $E$ whose diameter rel $W \leq \epsilon$ is at most $D(\chi(E), \epsilon)$ for any $\epsilon>0$. Also on every such surface there exists an essential simple closed curve of length $\leq B_{0}(E)$. Even more using a theorem of Freedman, Hass and Scott [7], we know that in every neighborhood of one of these simplicial hyperbolic surfaces, we can find an embedded level surface. Also by a theorem of 
Waldhausen [20], if two such embedded surfaces are disjoint then they enclose a submanifold of $V$ homeomorphic to $E \times[0,1]$.

Using the above information and for a given $\omega_{1}>0$ and if

$$
\omega / L \geq(2 n+5) \omega_{1} D\left(\chi(E), \epsilon_{1}\right),
$$

we can find $n$ essential closed curves $\bar{\alpha}_{1}, \ldots, \bar{\alpha}_{n}$ in $V$ and an embedding $\bar{\psi}: E \times$ $[0, n] \rightarrow V$ such that

- $\bar{\psi}$ induces a homotopy equivalence,

- each $\bar{\alpha}_{i}$ has length $\leq B_{0}(E)$ and is homotopic to a simple closed curve on $E$,

- $\bar{\alpha}_{i} \subset \bar{\psi}(E \times(i-1, i)), i=1, \ldots, n$,

- $\operatorname{diam}_{W}^{\geq \epsilon_{1}}(\bar{\psi}(E \times\{i\})) \leq D\left(\chi(E), \epsilon_{1}\right), i=0, \ldots, n$,

- $d_{W}^{\geq \epsilon_{1}}(\bar{\psi}(E \times\{i-1\}), \bar{\psi}(E \times\{i\}))$ is at least $\omega_{1}, i=1, \ldots, n$ and

- $d_{W}^{\geq \epsilon_{1}}\left(\bar{\alpha}_{i}, \bar{\psi}(E \times\{i-1\})\right)$ and $d_{W}^{\geq \epsilon_{1}}\left(\bar{\alpha}_{i}, \bar{\psi}(E \times\{i\})\right)$ are at least $\omega_{1}, i=1, \ldots, n$.

Now we consider the map $\psi=\phi^{-1} \circ \bar{\psi}: E \times[0, n] \rightarrow U$ and we have closed curves $\alpha_{1}=\phi\left(\bar{\alpha}_{1}\right), \alpha_{2}=\phi\left(\bar{\alpha}_{2}\right), \ldots, \alpha_{n}=\phi\left(\bar{\alpha}_{n}\right)$ such that for $\epsilon_{2}=\epsilon_{1} / L$ we have

- $\psi$ induces a homotopy equivalence,

- length of each $\alpha_{i}$ is at most $B_{0}(E) L$,

- $\alpha_{i} \subset \psi(S \times(i-1, i)), i=1, \ldots, n$,

- the diameter in $U_{\epsilon_{2}}$, rel $U^{\leq \epsilon_{2}}$, of each surface $\psi(E \times\{i\})$ is bounded by $L D\left(\chi(E), \epsilon_{1}\right), i=0, \ldots, n$,

- the distance in $U_{\epsilon_{2}}$, rel $U \leq \epsilon_{2}$, between $\psi(E \times\{i-1\})$ and $\psi(E \times\{i\})$ is at least $\omega_{1} / L$ and

- the distance in $U_{\epsilon_{2}}$, rel $U^{\leq \epsilon_{2}}$, between $\alpha_{i}$ and each of the surfaces $\psi(E \times\{i-1\})$ and $\psi(E \times\{i\})$ is at least $\omega_{1} / L, i=1, \ldots, n$.

One bothering feature of the above collection is that the lower bounds are obtained for distances rel $N^{\leq \epsilon_{2}}$ and in $N_{\epsilon_{2}}$ rather than for distances rel $N^{\leq \epsilon_{0}}$ and in $N_{\epsilon_{0}}$. This is however fixable using the next lemma:

Lemma 5.2 Given $0<\epsilon_{1}, \epsilon_{2}$ both smaller than the Margulis constant, there exists a constant $C=C\left(\epsilon_{1}, \epsilon_{2}\right) \geq 0$ depending only on $\epsilon_{1}$ and $\epsilon_{2}$ such that for every hyperbolic 3-manifold $N$ and $x, y \in N$

$$
\left.d_{N}^{\leq \epsilon_{1}}(x, y) \leq(C+1) d_{N}^{\leq \epsilon_{2}}(x, y)\right)+C .
$$


Proof If $\epsilon_{1} \geq \epsilon_{2}$ then obviously $N_{\epsilon_{1}} \subset N_{\epsilon_{2}}$ and

$$
d_{N}^{\leq \epsilon_{1}}(x, y) \leq d_{N}^{\leq \epsilon_{2}}(x, y)
$$

and we can let $C\left(\epsilon_{1}, \epsilon_{2}\right)=0$. So assume $\epsilon_{1}<\epsilon_{2}$.

By Lemma 2.1 one can see that if $T_{2}$ is a component of $N \leq \epsilon_{2}$ which contains a component $T_{1}$ of $N^{\leq \epsilon_{1}}$ then the distance from every point of $T_{2}$ to $T_{1}$ is bounded by a function of $\epsilon_{1}$ and $\epsilon_{2}$. In particular if the length of the core geodesic of a Margulis tube $T$ in $N \leq \epsilon_{2}$ is bigger than or equal to $\epsilon_{1}$ then the diameter of $T$ is bounded by a function of $\epsilon_{1}$ and $\epsilon_{2}$.

Let $g$ be a path connecting $x$ and $y$ which provides their distance rel $N^{\leq \epsilon_{2}}$, ie

$$
d^{\leq \epsilon_{2}}(x, y)=l(g)-l\left(g \cap N^{\leq \epsilon_{2}}\right),
$$

$g \subset N_{\epsilon_{2}}$ and $l\left(g \cap N^{\leq \epsilon_{2}}\right)$ denotes the sum of the lengths of the subsegments of $g$ inside components of $N^{\leq \epsilon_{2}}$. Assume $T_{2}$ is a component of $N \leq \epsilon_{2}$ with $g \cap T_{2} \neq \varnothing$ and $x^{\prime}, y^{\prime} \in \partial T_{2} \cap g$ are the entry and exit points of $g$. Obviously we are allowed to replace $g\left[x^{\prime}, y^{\prime}\right]$, the segment of $g$ connecting $x^{\prime}$ and $y^{\prime}$, with any other path inside $T_{2}$ that connects them. When the core curve of $T_{2}$ has length $\geq \epsilon_{1}$, ie $T \cap N^{<\epsilon_{1}}=\varnothing$, we assume $g\left[x^{\prime}, y^{\prime}\right]$ is the shortest geodesic connecting $x^{\prime}$ and $y^{\prime}$. Then it stays in $T_{2}$ (by convexity of $T_{2}$ ) and its length is bounded by the diameter of $T_{2}$ which is bounded by a function of $\epsilon_{1}$ and $\epsilon_{2}$ by our previous remark. If the core curve of $T_{2}$ has length $<\epsilon_{1}$, ie $T \cap N^{<\epsilon_{1}} \neq \varnothing$, let $T_{1} \subset T_{2}$ be the associated component of $N^{\leq \epsilon_{1}}$. In this case, we assume $g\left[x^{\prime}, y^{\prime}\right]$ is the concatenation of three segments $\alpha * \beta * \gamma$ where $\alpha$ is the shortest path connecting $x^{\prime}$ to $\partial T_{1}, \gamma$ is the shortest path connecting $y^{\prime}$ to $\partial T_{1}$ and $\beta$ is a path in $T_{1}$ that connects the endpoints of $\alpha$ and $\beta$ on $\partial T_{1}$. Again by our earlier remark, lengths of $\alpha$ and $\gamma$ are bounded by a function of $\epsilon_{1}$ and $\epsilon_{2}$ and as a result of all these

$$
l\left(g \cap N^{\leq \epsilon_{2}}\right)-l\left(g \cap N^{\leq \epsilon_{1}}\right) \leq C\left(\epsilon_{1}, \epsilon_{2}\right)\left|g \cap N^{\leq \epsilon_{2}}\right|
$$

where $C\left(\epsilon_{1}, \epsilon_{2}\right)$ is a constant depending only on $\epsilon_{1}$ and $\epsilon_{2}$ and the last quantity is the number of components of $N^{\leq \epsilon_{1}}$ which intersect $g$.

However by our assumption about the Margulis constant, the distance between every two components of $N \leq \epsilon_{2}$ is at least 1 and therefore

$$
d^{\leq \epsilon_{2}}(x, y)=l\left(g \cap N^{\leq \epsilon_{2}}\right) \geq\left|g \cap N^{\leq \epsilon_{2}}\right|-1 .
$$


Now using (5-1), (5-2), (5-3) and the fact that $g \subset N_{\epsilon_{2}} \subset N_{\epsilon_{1}}$ we have

$$
\begin{aligned}
d_{N}^{\leq \epsilon_{1}}(x, y) & \leq l(g)-l\left(g \cap N^{\leq \epsilon_{1}}\right) \\
& \leq l(g)-l\left(g \cap N^{\leq \epsilon_{2}}\right)+C\left(\epsilon_{1}, \epsilon_{2}\right)\left|g \cap N^{\leq \epsilon_{1}}\right| \\
& \leq d^{\leq \epsilon_{2}}(x, y)+C\left(\epsilon_{1}, \epsilon_{2}\right)\left(d^{\leq \epsilon_{2}}(x, y)+1\right) \\
& =\left(C\left(\epsilon_{1}, \epsilon_{2}\right)+1\right) d^{\leq \epsilon_{2}}(x, y)+C\left(\epsilon_{1}, \epsilon_{2}\right)
\end{aligned}
$$

which finishes the proof of the lemma.

Using this lemma we can replace the lower bound $\omega_{1} / L$ for the distances rel $N^{\epsilon_{2}}$ with the lower bound $\omega_{2}=(C+1)\left(\omega_{1} / L\right)+C$ for the distances rel $N \leq \epsilon_{0}$ and $C=C\left(\epsilon_{1}, \epsilon_{2}\right)$. Since $\epsilon_{2}=\epsilon_{0} / L^{2}$ this depends only on our choice of $\omega_{1}, \epsilon_{0}$ and $L$. Besides it tends to infinity as $\omega_{1} \rightarrow \infty$.

By assuming that $\omega$ is large, we can make sure that the above collection of closed curves is constructed in a way that its distance to $\partial U$ is at least $B_{0}(E) L$. Then it follows from Lemma 3.9 that each $\alpha_{i}, i=1, \ldots, n$, is homotopically nontrivial in $N$. Even more the geodesic representatives of $\alpha_{1}, \ldots, \alpha_{n}$ in $N$, which we respectively denote by $\alpha_{1}^{*}, \ldots, \alpha_{n}^{*}$, are within $U$ and by Lemma 2.1

$$
d_{N}^{\leq \epsilon_{0}}\left(\alpha_{i}, \alpha_{i}^{*}\right) \leq c_{1}=\cosh ^{-1}\left(B_{0}(E) L / \epsilon_{0}\right), \quad i=1, \ldots, n .
$$

The length of each of the curves $\alpha_{i}^{*}$ is still bounded from above by $B_{0}(E) L$. Next we want to change each curve $\alpha_{i}^{*}$ to guarantee that $\operatorname{rad}\left(\alpha_{i}^{*}\right) \geq 0.025$ for every $i$, where $\operatorname{rad}(\gamma)$ denotes the tube radius of $\gamma$. Meyerhoff [14] defines a monotonically decreasing function $r:(0,0.1] \rightarrow[0.3, \infty)$ such that if $\gamma$ is a closed geodesic in $N$ and $l_{N}(\gamma) \leq t, \operatorname{rad}(\gamma) \geq r(t)$. So if $l_{N}\left(\alpha_{i}^{*}\right) \leq 0.1$ then $\operatorname{rad}\left(\alpha_{i}^{*}\right) \geq 0.3$ and we are satisfied with its tube radius.

Following Gabai [8] assume $l_{N}\left(\alpha_{i}^{*}\right) \geq 0.1$ and $\operatorname{rad}\left(\alpha_{i}^{*}\right) \leq 0.025 \leq \frac{1}{4} l_{N}\left(\alpha_{i}^{*}\right)$ then there exists a geodesic $\beta_{i}^{*}$ homotopic to a curve which is a union of a segment of $\alpha_{i}^{*}$ and an orthogonal arc from $\alpha_{i}^{*}$ to itself, and each of these segments has length $\leq l_{N}\left(\alpha_{i}^{*}\right) / 2$. By straightening these segments and using the law of cosines, we see that since $l\left(\alpha_{i}^{*}\right) \geq 0.1, l\left(\beta_{i}^{*}\right)<l\left(\alpha_{i}^{*}\right)-0.02$. Note that

$$
d_{N}^{\leq \epsilon_{0}}\left(\alpha_{i}^{*}, \beta_{i}^{*}\right) \leq c_{1}+0.025
$$

by another application of Lemma 2.1. If $\operatorname{rad}\left(\beta_{i}\right) \leq .025$ then we can repeat the same procedure and obtain a shorter closed geodesic and repeat the same procedure if necessary. However such a process reduces the length by at least 0.02 and since the length of the original curve was bounded from above by $B_{0}(E) L$ the process must 
stop after at most $B_{0}(E) L / 0.02$ times. At the end of the process we will be left with a closed geodesic $\gamma_{i}$ with $\operatorname{rad}\left(\gamma_{i}\right) \geq 0.025$.

As we have seen before, the distance rel $N \leq \epsilon_{0}$ between two closed geodesics that appear consecutively is at most $c_{1}+0.025$ and the length of each of them is bounded from above by $B_{0}(E) L$ and therefore

$$
d_{N}^{\leq \epsilon_{0}}\left(\alpha_{i}, \gamma_{i}\right) \leq c_{2}=\left(\frac{B_{0}(E) L}{0.02}+1\right)\left(c_{1}+0.025+B_{0}(E) L\right)
$$

for every $1 \leq i \leq n$. So provided that

$$
\omega_{2}>c_{2}+B_{0}(E) L+D\left(\chi(E), \epsilon_{0}\right)+1
$$

for every $i=1, \ldots, n$, the geodesic $\gamma_{i}$ will stay in $\psi(E \times(i-1, i))$. Also the distance, rel $N^{\leq \epsilon_{0}}$, between $\psi(E \times\{i-1\})$ and $\psi(E \times\{i\})$ is at least $D\left(\chi(E), \epsilon_{0}\right)$ and the distance between $\gamma_{i}$ and each of the surfaces $\psi(E \times\{i-1\})$ and $\psi(E \times\{i\})$ is at least 1 . We already know $\psi$ induces a homotopy equivalence between $E \times[0, n]$ and $U$ and the diameter, rel $N^{\leq \epsilon_{0}}$, of each surface $\psi(E \times\{i\})$ is at most $G_{\epsilon_{0}, \epsilon_{2}}\left(L D\left(\chi(E), \epsilon_{1}\right)\right)$. Hence Proposition 5.1 follows.

Once we know Proposition 5.1, we turn our attention to proving the main result of this section and as a corollary prove Theorem 1.2 and Theorem 1.3. First we consider the especial case when $(M, P)=(\bar{S} \times I, \partial \bar{S} \times I)$ for a compact surface of finite type. Note that given a proper embedding $j: M \backslash P \rightarrow N$ of a pared manifold $(M, P)$, a free side $E$ and a product region $U$ of $N$, we say $j(E)$ is a level surface of $U$ if $U$ is disjoint from the image of $j$ and the $j$ image of $E$ and a level surface of $U$ bound an interval bundle whose interior is disjoint from the image of $j$.

Theorem 5.3 Given a compact surface of finite type $\bar{S}$ with interior $S$ and constants $\epsilon_{0}, L$, there is a constant $\omega$ such that the following holds. Suppose $j: S \times I \rightarrow N$ is a proper embedding of the pared manifold $(\bar{S} \times I, \partial \bar{S} \times I)$ into the oriented hyperbolic manifold $N$ and the $j$ images of each free side is either a level surface of an $L$-product region of $\epsilon_{0}-$ width $\omega$ or is incompressible to the outside. Then there exists a $0.025-$ separated geodesic link $\Gamma$ in the product regions such that $j$ is 2 -incompressible rel $\Gamma$.

Even more length of each component of $\Gamma$ is bounded depending on $\chi(S)$ and $L$ and the number of components of $\Gamma$ only depends on $\chi(S)$.

Proof First of all note that we can assume the map induced by $j$ on the fundamental groups is surjective. Otherwise, we can consider the cover associated to the image of 
$\pi_{1}(S \times I)$; then $j$ lifts to an embedding $\widetilde{j}$ and every product region associated to a free side, lifts isometrically to a product region for the same free side. Suppose $\widetilde{\Gamma}$ is a geodesic link in the product regions of the cover and $\tilde{j}$ is 2 -incompressible rel $\widetilde{\Gamma}$; then $\widetilde{\Gamma}$ maps isometrically to a geodesic link $\Gamma$ with the same separation constant in $N$ and it should be clear that $j$ is also 2 -incompressible rel $\Gamma$. Using this observation, we can, in particular, assume the image of $j$ separates $N$. We also identify $S$ with the middle surface of $S \times I$. Note that as we pointed out earlier, given a geodesic link in the complement of the image of $j, 2$-incompressibility of the map $j$ as a proper embedding of a pared manifold is equivalent to 2-incompressibility of $j(S)$ as a properly embedded surface of finite type.

Let $E^{+}$and $E^{-}$respectively denote the top and bottom free sides of $(\bar{S} \times I, \partial \bar{S} \times I)$ with the induced orientations. In particular $E^{+}$and $E^{-}$are homeomorphic to $S$ but $E^{-}$has the opposite orientation. If the $j$-images of $E^{+}$and $E^{-}$are both incompressible to the outside, then they are incompressible and $j$ is obviously $2-$ incompressible rel $\Gamma=\varnothing$. We mainly deal with the case when the $j$-image of neither $E^{+}$nor $E^{-}$in incompressible to the outside and are level surfaces of $L$-product regions $U^{+}, U^{-}$(respectively) of $\epsilon_{0}$-width $\omega$. At the end, we explain what happens if one of them is incompressible to the outside.

Let $n=3|\chi(S)|+1$. By Proposition 5.1, if $\omega$ is sufficiently large, we have geodesic links $\Gamma^{+}=\left\{\gamma_{1}^{+}, \ldots, \gamma_{n}^{+}\right\} \subset U^{+}, \Gamma^{-}=\left\{\gamma_{1}^{-}, \ldots, \gamma_{n}^{-}\right\} \subset U^{-}$and embeddings $\psi^{+}: E^{+} \times[0, n] \rightarrow U^{+}, \psi^{-}: E^{-} \times[0, n] \rightarrow U^{-}$which satisfy the conclusion of the proposition. (At this point the map $\psi^{+}$is orientation preserving but $\psi^{-}$is orientation reversing as maps from $S \times[0, n]$ to $N$, since $E^{+}$and $E^{-}$are quipped with different orientations on $S$.)

We claim $j$ is 2 -incompressible rel $\Gamma=\Gamma^{+} \cup \Gamma^{-}$. This proves the theorem in this case, since the tube radii of elements of $\Gamma$ are bounded below by 0.025 , the distance between every two of them is bounded from below. In addition the length of each component of $\Gamma$ is bounded from above by $L B_{0}(S)$ and the number of components is a function of $\chi(S)$.

We say a proper map $f: R \rightarrow N$ from a surface $R$ (or its image $f(R)$ ) separates subsets $A, B \subset N$ if for every arc $\kappa:[0,1] \rightarrow N$ that connects $A$ and $B$, ie $\kappa(0) \in$ $A, \kappa(1) \in B$, we have $\langle f(R), \kappa\rangle \neq 0$ where $\langle\cdot, \cdot\rangle$ denotes the algebraic intersection number. Obviously $j \mid E^{+}$and also $j \mid E^{-}$separate every $\gamma_{i}^{+}$from every $\gamma_{m}^{-}, i, m=$ $1, \ldots, n$.

If $j(S)$ is not 2-incompressible rel $\Gamma$, then either the $j$ image of a noncontractible loop of $S$ is homotopic in $N \backslash \Gamma$ to a multiplied meridian of a component of $\Gamma$ or the $j$-image of a simple nonperipheral loop of $S$ is freely homotopic (in $N \backslash \Gamma$ ) into a 
cusp of $N$. (Note that from now on even when the $j$-image of a loop is contractible in $N \backslash \Gamma$, we say it is freely homotopic, in $N \backslash \Gamma$, to a multiplied meridian of a component of $\Gamma$.) Suppose the $j$-image of a noncontractible loop of $S$ is freely homotopic, in $N \backslash \Gamma$, to a multiplied meridian of a component of $\Gamma$. Since $j(S)$ is embedded, an easy application of the Loop Theorem shows there exists an embedding $D^{2} \hookrightarrow N$ whose image intersects $j(S)$ in the image of $\partial D^{2}$ which is the $j$-image of a noncontractible simple loop on $S$ and this disk either misses $\Gamma$ or intersects $\Gamma$ at most once. In other terms, this provides a compression of $j(S)$ that either misses $\Gamma$ or crosses $\Gamma$ at most once at $\alpha_{1} \in \Gamma$. If possible compress again via a compression meeting $\Gamma \backslash\left\{\alpha_{1}\right\}$ at most once, say at $\alpha_{2} \in \Gamma$. After at most $|\chi(S)|$ such compressions and deleting 2 -spheres, we are left with a collection of properly embedded connected surfaces. Let $R$ be one of these properly embedded surfaces; then we know $R$ is not a 2-sphere and a noncontractible loop on $R$ is not freely homotopic, in $N \backslash \Gamma_{1}$, to a multiplied meridian of a component of $\Gamma_{1}$, where $\Gamma_{1}=\Gamma \backslash\left\{\alpha_{1}, \ldots, \alpha_{s}\right\}(s \leq|\chi(S)|)$. In particular $R$ is incompressible in $N \backslash \Gamma_{1}$. An easy application of the Annulus Theorem shows that if there is a nonperipheral loop on $R$ which is freely homotopic (within $N \backslash \Gamma_{1}$ ) into a cusp of $N$, then there is a proper embedding $S^{1} \times[0, \infty) \hookrightarrow N$ that does not intersect $\Gamma, S^{1} \times\{0\}$ is a nonperipheral loop on $R$ and $S^{1} \times\{t\}$ is in a neighborhood of a cusp of $N$ for $t$ sufficiently large. Using this annulus we can perform a surgery (in $N \backslash \Gamma_{1}$ ) on $R$ and obtain new properly embedded surface(s). We call such an operation an annular surgery. If possible we perform more such annular surgeries, in $N \backslash \Gamma_{1}$, for the new properly embedded surfaces and other surfaces in the collection and remove all the 2 -spheres. The total number of compressions and surgeries like the ones described above will be at most $|\chi(S)|$ and we will be left with properly embedded connected surfaces $S_{1}, \ldots, S_{r}(r \leq|\chi(S)|)$, none of which is a 2 -sphere and each is 2-incompressible rel $\Gamma_{1}$.

Since $n=3|\chi(S)|+1 \geq 2 \chi(S)+1+s, \Gamma^{+} \cap \Gamma_{1}$ and $\Gamma^{-} \cap \Gamma_{1}$ are nonempty and each has at least $2|\chi(S)|+1$ elements. Even more each $\gamma_{i}^{+} \in \Gamma^{+} \cap \Gamma_{1}$ is separated from each $\gamma_{m} \in \Gamma^{-} \cap \Gamma_{1}$ by exactly one of the surfaces $S_{1}, \ldots, S_{r}$. Using this, it is easy to see there is one of the surfaces, say $S_{1}$, which separates 3 distinct components of $\Gamma^{+} \cap \Gamma_{1}$ from 3 distinct components of $\Gamma^{-} \cap \Gamma_{1}$. In particular, we can choose $i, m, k, l$ such that $S_{1}$ separates $\gamma_{i}^{+}, \gamma_{k}^{+} \in \Gamma_{1}$ from $\gamma_{m}^{-}, \gamma_{l}^{-} \in \Gamma_{1}$ with $i+2<k$ and $m+2<l$.

Now we use Theorem 3.7 to construct a simplicial hyperbolic surface $\Sigma_{1}$ which is $\Gamma_{1}-$ homotopic to $S_{1}$. Note that $\Sigma_{1}$ also separates $\gamma_{i}^{+}, \gamma_{k}^{+}$from $\gamma_{m}^{-}, \gamma_{l}^{-}$. Let $Z_{1}$ be the region homeomorphic to $S \times I$ enclosed by $\psi^{+}\left(E^{+} \times\{i+2\}\right)$ and $\psi^{-}\left(E^{-} \times\{m+2\}\right)$. Also let $Z_{2} \subset Z_{1}$ be the region homeomorphic to $S \times I$ enclosed by $\psi^{+}\left(E^{+} \times\{i+1\}\right)$ and $\psi^{-}\left(E^{-} \times\{m+1\}\right)$. Obviously $j(S \times I) \subset Z_{2} \subset Z_{1}$. 
The region $Z_{2}$ is connected and contains $\gamma_{i}^{+}$and $\gamma_{m}^{-}$and since $\Sigma_{1}$ separates these two, $\Sigma_{1}$ must intersect $Z_{2}$. On the other hand, the diameter of $\Sigma_{1}$, rel $N^{\leq \epsilon_{0}}$, is at most $D\left(\chi(S), \epsilon_{0}\right)$ and properties of $\psi^{+}$and $\psi^{-}$imply that the distances, rel $N^{\leq \epsilon_{0}}$, between $Z_{2}$ and boundary components of $Z_{1}$ are at least $D\left(\chi(S), \epsilon_{0}\right)$. Hence $\Sigma_{1} \subset Z_{1}$.

But $\gamma_{k}^{+}$and $\gamma_{l}^{-}$do not intersect $Z_{1}$, and so we can connect them to $\psi^{+}\left(E^{+} \times\{n\}\right)$ and $\psi^{-}\left(E^{-} \times\{n\}\right)$ without intersecting $\Sigma_{1}$. This shows that $\Sigma_{1}$ separates $\psi^{+}\left(E^{+} \times\{n\}\right)$ and $\psi^{-}\left(E^{-} \times\{n\}\right)$. Thus $\left\langle\kappa, \Sigma_{1}\right\rangle \neq 0$ for every arc $\kappa$ that connects $\psi^{+}\left(E^{+} \times\{n\}\right)$ and $\psi^{-}\left(E^{-} \times\{n\}\right)$. The region $X$ enclosed by $\psi^{+}\left(E^{+} \times\{n\}\right)$ and $\psi^{-}\left(E^{-} \times\{n\}\right)$ is homeomorphic to $S \times I$ and it follows that the restriction to $\Sigma_{1}$ of the projection from $X$ to $S$ induces a nonzero degree map from $\Sigma_{1}$ to $S$ and we must have $\chi\left(\Sigma_{1}\right) \leq \chi(S)$. This is possible only when we have done no compression and no annular surgery on $j(S)$. In other terms $j(S)$ is 2-incompressible rel $\Gamma$.

We are now left with the case when the $j$-image of one of the surfaces $E^{+}, E^{-}$, say $E^{-}$, is incompressible to the outside and $j\left(E^{+}\right)$is a level surface of an $L$-product region of $\epsilon_{0}$-width $\omega$, where $\omega$ is chosen as above. In this case, we let $\Gamma^{-}=\varnothing$ and we choose $\Gamma=\Gamma^{+}$as before. Let $N^{\prime}$ denote the 3-manifold obtained by removing the interiors of cusps of $N$ that are associated to boundary components of $\bar{S}$. Then our observation and assumption in the beginning of the proof, that $j$ induces a surjective map on the level of fundamental groups, tells us that $N^{\prime}$ has two ends associated to $E^{+}$and $E^{-}$; we call them the $E^{+}-$end and the $E^{-}-$end respectively. In addition $j(S) \cap N^{\prime}$ "separates" these two ends. In a similar fashion to what we defined earlier, we say a proper map $f: R \rightarrow N$ from a surface $R$ to $N$ separates a subset $A$ from the $E^{-}$-end of $N^{\prime}$, if given an arc $\kappa:[0, \infty) \rightarrow N^{\prime}$ that "connects" them, ie $\kappa(0) \in A$ and $\kappa(t)$ exits the $E^{-}$-end as $t \rightarrow \infty,\left\langle R \cap N^{\prime}, \kappa([0, \infty))\right\rangle \neq 0$. Then obviously $j(S)$ separates every $\gamma_{i}^{+}$from the $E^{-}$-end of $N^{\prime}$. If $j(S)$ is not 2-incompressible rel $\Gamma$, after at most $|\chi(S)|$ compressions and annular surgeries like above, we are left with a collection of properly embedded nonspherical surfaces $S_{1}, \ldots, S_{r}(r \leq|\chi(S)|)$ and a subcollection $\Gamma_{1} \subset \Gamma$ of at least $2|\chi(S)|+1$ closed geodesics that were not touched by the compressions. Every element of $\Gamma_{1}$ is separated from the $E^{-}$-end of $N^{\prime}$ by one of the surfaces $S_{1}, \ldots, S_{r}$ and in particular one of them, say $S_{1}$, separates three distinct elements of $\Gamma_{1}$ from the $E^{-}$-end of $N^{\prime}$. The surface $S_{1}$ is 2-incompressible rel $\Gamma_{1}$ and after shrinkwrapping, we find a simplicial hyperbolic surface $\Sigma_{1}$ which is $\Gamma_{1}$-homotopic to $S_{1}$. An argument similar to the above shows that $\Sigma_{1}$ separates $\psi^{+}\left(E^{+} \times\{n\}\right)$ from the $E^{-}$-end of $N$. Then it follows that there is a nonzero degree map from $\Sigma_{1}$ to $S$ and therefore $\chi\left(\Sigma_{1}\right) \leq \chi(S)$. This will be possible only when we have had no compression or annular surgery and $j(S)$ is 2 -incompressible rel $\Gamma$. $\square$

Note that the above theorem obviously implies Theorem 1.3. We use the above theorem to prove our main theorem for general pared manifolds and as a consequence 
Theorem 1.2. Given a proper embedding $j: M \backslash P \rightarrow N$ of a pared manifold $(M, P)$ into a hyperbolic 3-manifold $N$, we say the $j$-image of a free side $E$ has no accidental parabolics if the $j$-image of a nonperipheral simple loop on $E$ is not freely homotopic into a cusp of $N$.

Theorem 5.4 Given a pared manifold $(M, P)$ with no torus boundary, constants $\epsilon_{0}$ and $L$, there is a constant $\omega$ such that the following holds. Suppose $j: M \backslash P \rightarrow N$ is a proper embedding of $(M, P)$ into an oriented hyperbolic 3-manifold $N$ and the $j$-image of each free side $E$ of $(M, P)$ is either a level surface of an $L$-product region of $\epsilon_{0}$-width $\omega$ or $E$ is incompressible, $j(E)$ is incompressible to the outside and has no accidental parabolics. Then there exists a 0.025 -separated geodesic link $\Gamma$ in the product regions associated to free sides of $(M, P)$ such that $j$ is 2-incompressible rel $\Gamma$.

Even more length of each component of $\Gamma$ is bounded depending on the topology of free sides of $(M, P)$ and $L$ and the number of components of $\Gamma$ depends only on the topology of free sides of $(M, P)$.

Proof Using Theorem 5.3 we know that if $\omega$ is sufficiently large, and we choose a level surface $E^{\prime}$ in the middle of a product region associated to a free side $E$ of $(M, P)$ then there is a 0.025 -separated geodesic link $\Gamma_{E}$ in this product region such that $E^{\prime}$ is 2-incompressible rel $\Gamma_{E}$. For every free side $E$ of $(M, P)$ let $E^{\prime}=E$ and $\Gamma_{E}=\varnothing$, if $E$ is incompressible, $j(E)$ is incompressible to the outside and has no accidental parabolics; otherwise let $E^{\prime}$ be the level surface that we just described. Also let $\Gamma$ be the geodesic link that is the union of $\Gamma_{E}$ over all free sides of $(M, P)$. We claim $j$ is 2 -incompressible rel $\Gamma$.

We define $X$ to be the region in $N$ that is enclosed by the surfaces $E^{\prime}$ for all free sides $E$ of $(M, P)$. Obviously $X$ is a collared neighborhood of $j(M \backslash P)$ and is homeomorphic to $M \backslash P$. Assume there is a noncontractible loop in $M$ whose $j-$ image is freely homotopic (in $N \backslash \Gamma$ ) to a multiplied meridian of a component of $\Gamma$. Then there is a map $g: D^{2} \rightarrow N$ where the image of $\partial D^{2}$ is the $j$-image of a noncontractible loop of $M$ and the preimage of $\Gamma$ is at most a single point on $D^{2}$. We assume the map $g$ is chosen in a way that $g\left(D^{2}\right)$ intersects $\partial X$ transversally and the number of components of $g^{-1}(\partial X)$ is the minimum among such maps. In particular every component of $g\left(D^{2}\right) \cap \partial X$ will be essential on $\partial X$. We claim $g\left(D^{2}\right)$ cannot intersect a component of $\partial X$ that is 2-incompressible rel $\Gamma$. Otherwise the intersection would provide an essential loop on that component of $\partial X$ which would be homotopic (in $N \backslash \Gamma$ ) to a multiplied meridian of a component of $\Gamma$ and this would be a contradiction. We knew that other components of $\partial X$ are $j$-images of incompressible 
free sides of $(M, P)$, whose $j$-images are incompressible to the outside. The image of an innermost loop of $g^{-1}(\partial X)$ is on one such component of $\partial X$. However this loop will bound a disk outside or inside $X$ which is impossible unless the loop is contractible on $\partial X$ and we have a contradiction. So $g\left(D^{2}\right)$ cannot intersect $\partial X$ but then this implies that $g\left(\partial D^{2}\right)$ is contractible in $X$ and therefore is contractible in $j(M \backslash P)$.

On the other hand assume there is a nonperipheral simple loop on a free side of $(M, P)$ whose $j$-image is freely homotopic (within $N \backslash \Gamma$ ) into a cusp of $N$. We already know that the $j$-image of every free side is incompressible in $N \backslash \Gamma$ and therefore using the Annulus Theorem, we can assume there is an embedding $S^{1} \times[0, \infty) \hookrightarrow N$ where $S^{1} \times\{0\}$ is the $j$-image of a nonperipheral simple loop on a free side of $(M, P)$, $S^{1} \times\{t\}$ is in a neighborhood of a cusp of $N$ for $t$ sufficiently large and $S^{1} \times(0, \infty)$ does not intersect the $j$-images of free sides of $(M, P)$. Then it should be easy to see that we can deform the embedding $S^{1} \times[0, \infty) \hookrightarrow N$ in a way that it does not intersect $\partial X$. This is again by using the fact that components of $\partial X$ are either $j-$ images of free sides of $(M, P)$ or are 2 -incompressible rel $\Gamma$. We know the $j$-image of a free side of $(M, P)$, which is not 2-incompressible, has no accidental parabolics and therefore the cylinder $S^{1} \times[0, \infty)$ must be inside the image of $j$ and $S^{1} \times\{0\}$ is the $j$-image of a peripheral loop in $(M, P)$. This contradicts our assumption.

\section{References}

[1] B H Bowditch, Markoff triples and quasi-Fuchsian groups, Proc. London Math. Soc. (3) 77 (1998) 697-736 MR1643429

[2] J Brock, R D Canary, Y N Minsky, The classification of Kleinian surface groups II: the ending lamination conjecture arXiv:math/0412006 1

[3] J Brock, Y N Minsky, H Namazi, J Souto, Splittings, models and bounds in hyperbolic geometry, in preparation

[4] R Brooks, J P Matelski, Collars in Kleinian groups, Duke Math. J. 49 (1982) 163-182 MR650375

[5] D Calegari, D Gabai, Shrinkwrapping and the taming of hyperbolic 3-manifolds, J. Amer. Math. Soc. 19 (2006) 385-446 MR2188131

[6] RD Canary, A covering theorem for hyperbolic 3-manifolds and its applications, Topology 35 (1996) 751-778 MR1396777

[7] M Freedman, J Hass, P Scott, Least area incompressible surfaces in 3-manifolds, Invent. Math. 71 (1983) 609-642 MR695910

[8] D Gabai, On the geometric and topological rigidity of hyperbolic 3-manifolds, J. Amer. Math. Soc. 10 (1997) 37-74 MR1354958 
[9] D Gabai, Hyperbolic geometry and 3-manifold topology, from: "Low dimensional topology”, (T S Mrowka, P S Ozsváth, editors), IAS/Park City Math. Ser. 15, Amer. Math. Soc. (2009) 73-103 MR2503493

[10] S Kojima, Deformations of hyperbolic 3-cone-manifolds, J. Differential Geom. 49 (1998) 469-516 MR1669649

[11] H A Masur, Y N Minsky, Geometry of the complex of curves. I. Hyperbolicity, Invent. Math. 138 (1999) 103-149 MR1714338

[12] H A Masur, Y N Minsky, Geometry of the complex of curves. II. Hierarchical structure, Geom. Funct. Anal. 10 (2000) 902-974 MR1791145

[13] H A Masur, Y N Minsky, Quasiconvexity in the curve complex, from: "In the tradition of Ahlfors and Bers, III", (W Abikoff, A Haas, editors), Contemp. Math. 355, Amer. Math. Soc. (2004) 309-320 MR2145071

[14] R Meyerhoff, A lower bound for the volume of hyperbolic 3-manifolds, Canad. J. Math. 39 (1987) 1038-1056 MR918586

[15] Y N Minsky, The classification of Kleinian surface groups I: models and bounds, to appear in Ann. of Math. (2) arXiv:math/0302208v3

[16] Y N Minsky, Bounded geometry for Kleinian groups, Invent. Math. 146 (2001) 143-192 MR1859020

[17] H Namazi, Heegaard splittings with bounded combinatorics, in preparation

[18] H Namazi, J Souto, Revisiting Thurston's uniform injectivity theorem, in preparation

[19] T Soma, Existence of ruled wrappings in hyperbolic 3-manifolds, Geom. Topol. 10 (2006) 1173-1184 MR2255495

[20] F Waldhausen, On irreducible 3-manifolds which are sufficiently large, Ann. of Math. (2) 87 (1968) 56-88 MR0224099

Department of Mathematics, University of Texas at Austin

1 University Station C1200, Austin, TX 78712

hossein@math .utexas .edu

http://ma.utexas.edu/users/hossein/

Received: 12 January 2009 Revised: 27 September 2009 